\title{
COMPLEXITY PROBLEMS IN ENUMERATIVE COMBINATORICS
}

\author{
IGOR PAK ${ }^{\star}$
}

\begin{abstract}
We give a broad survey of recent results in Enumerative Combinatorics and their complexity aspects.
\end{abstract}

\section{INTRODUCTION}

The subject of enumerative combinatorics is both classical and modern. It is classical, as the basic counting questions go back millennia; yet it is modern in the use of a large variety of the latest ideas and technical tools from across many areas of mathematics. The remarkable successes from the last few decades have been widely publicized; yet they come at a price, as one wonders if there is anything left to explore. In fact, are there enumerative problems that cannot be resolved with existing technology? In this paper we present many challenges in the field from the computational complexity point of view, and describe how recent results fit into the story.

Let us first divide the problems into three major classes. This division is not as neat as it may seem, as there are problems which fit into multiple or none of the classes, especially if they come from other areas. Still, it provides us with a good starting point.

(1) Formulas. Let $\mathcal{P}$ be a set of combinatorial objects - think of trees, words, permutations, Young tableaux, etc. Such objects often come with a parameter $n$ corresponding to the size of the objects. Let $\mathcal{P}_{n}$ be the set of objects of size $n$. Find a formula for $\left|\mathcal{P}_{n}\right|$.

(2) Bijections. Now let $\mathcal{P}$ and $\mathcal{Q}$ be two sets of (possibly very different) combinatorial objects. Suppose that you know (or at least suspect) that $\left|\mathcal{P}_{n}\right|=\left|\mathcal{Q}_{n}\right|$. Find an explicit bijection $\varphi: \mathcal{P}_{n} \rightarrow \mathcal{Q}_{n}$.

(3) Combinatorial interpretations. Now suppose there is an integer sequence $\left\{a_{n}\right\}$ given by a formula. Suppose that you know (or at least suspect) that $a_{n} \geq 0$ for all $n$. Find a combinatorial interpretation of $a_{n}$, i.e. a set of combinatorial objects $\mathcal{P}$ such that $\left|\mathcal{P}_{n}\right|=a_{n}$.

People in the area are well skilled in both resolving and justifying these problems. Indeed, a formula is a good thing to have in case one needs to compute $\left|\mathcal{P}_{n}\right|$ explicitly for large $n$, find the asymptotics, gauge the structural complexity of the objects, etc. A bijection between a complicated set $\mathcal{P}$ and a simpler set $\mathcal{Q}$ is an even better thing to have, as it allows one to better understand the nature of $\mathcal{P}$, do a refined counting of $\mathcal{P}_{n}$ with respect to various statistics, generate elements of $\mathcal{P}_{n}$ at random, etc. Finally, a combinatorial interpretation is an excellent first step that allows one to proceed to (1) and then (2), or at least obtain some useful estimates for $a_{n}$.

Here is a more difficult part, which comes in the form of inquisitive questions in each case: $\left(1^{\prime}\right)$ What is a formula? What happens if there is no formula? Can you prove there isn't one? How do you even formalize the last question if you don't know the answer to the first?

April 3, 2018.

*Department of Mathematics, UCLA, Los Angeles, CA 90095. Email: pak@math.ucla.edu. 
$\left(2^{\prime}\right)$ There are, obviously, $\left|\mathcal{P}_{n}\right|$ ! bijections $\varphi: \mathcal{P}_{n} \rightarrow \mathcal{Q}_{n}$, so you must want a particular one, or at least one with certain properties? Is there a "canonical" bijection, or at least the one you want the most? What if there isn't a good bijection by whatever measure - can you prove that? Can you even formalize that?

$\left(3^{\prime}\right)$ Again, what do you do in the case when there isn't a combinatorial interpretation? Can you formally prove a negative result so that others stop pursuing these problems?

We give some formal answers to these questions, at least in several interesting special cases. As the reader will see, the complexity approach brings some clarity in most cases. But to give the answers we first need to explain the nature of combinatorial objects in each case, and to review the literature. That is the goal of this survey.

Before we conclude the introduction, let us quote Gian-Carlo Rota, one of the founding fathers of modern enumerative combinatorics:

"Combinatorics is an honest subject. No adèles, no sigma-algebras. You count balls in a box, and you either have the right number or you haven't. You get the feeling that the result you have discovered is forever, because it's concrete. Other branches of mathematics are not so clear-cut, [..] never fully convincing: you don't get a feeling of having done an honest day's work. Don't get the wrong idea-combinatorics is not just putting balls into boxes. Counting finite sets can be a highbrow undertaking, with sophisticated techniques." [154]

Rota is right, of course, historically speaking. When the result is positive, it's "forever" indeed, and this partly explains the glamour of (1) - (3). But when the result is negative, when questions $\left(1^{\prime}\right)-\left(3^{\prime}\right)$ are addressed, this certainty disappears. Our current understanding of a "formula" and a "good bijection" can change in the future, perhaps fundamentally, as it has changed in the past. Forever these results are certainly not. In fact, when the complexity assumptions such as $\mathrm{P} \neq \mathrm{NP}, \mathrm{FP} \neq \# \mathrm{P}$, etc. become essential, one must learn to accept the uncertainty and learn to navigate in this new environment. . at least until computational complexity brings more clarity to these matters.

\section{What is A FORMUla?}

1.1. Basic examples. We start with the Fibonacci numbers [158, A000045]:

$$
\begin{gathered}
F_{n}=F_{n-1}+F_{n-2}, \quad F_{0}=F_{1}=1 \\
F_{n}=\sum_{i=0}^{\lfloor n / 2\rfloor}\left(\begin{array}{c}
n-i \\
i
\end{array}\right) \\
F_{n}=\frac{1}{\sqrt{5}}\left(\phi^{n}-(-\phi)^{-n}\right), \quad \text { where } \phi=\frac{1+\sqrt{5}}{2} \\
F_{n}=\left(A^{n}\right)_{2,2}, \quad \text { where } A=\left(\begin{array}{ll}
0 & 1 \\
1 & 1
\end{array}\right) .
\end{gathered}
$$

Equation (1.1) is usually presented as a definition, but can also be used to compute $F_{n}$ in poly $(n)$ time. Equation (1.2) is useful to place Fibonacci numbers in the hierarchy of integer sequences (see below). Equation (1.3) is useful to obtain asymptotics, and equation (1.4) 
gives a fast algorithm for computing $F_{n}$ (by repeated squaring). The moral: there is no one notion of a "good formula", as different equations have different uses.

Let us consider a few more plausible formula candidates:

$$
D_{n}=[[n ! / e]] \text {, where }[[x]] \text { denotes the nearest integer }
$$

$$
\begin{gathered}
C_{n}=\left[t^{n}\right] \frac{1-\sqrt{1-4 t}}{2 t} \\
E_{n}=n ! \cdot\left[t^{n}\right] y(t), \quad \text { where } 2 y^{\prime}=1+y^{2}, y(0)=1 \\
T_{n}=(n-1) ! \cdot\left[t^{n}\right] z(t), \quad \text { where } z=t e^{t e^{t e^{t e}}} .
\end{gathered}
$$

Here $D_{n}$ is the number of derangements (fixed-point-free permutations in $S_{n}$ ), $C_{n}$ is the Catalan number (the number of binary trees with $n$ vertices), $E_{n}$ is the Euler number (the number of alternating permutations $\sigma(1)<\sigma(2)>\sigma(3)<\sigma(4)>\ldots$ in $S_{n}$ ), and $T_{n}$ is the Cayley number (the number of spanning trees in the complete graph $K_{n}$ ). Here and everywhere below we use $\left[t^{n}\right] F(t)$ to denote the coefficient of $t^{n}$ in $F(t)$.

Note that in each case above, there are better formulas for applications:

$$
\begin{gathered}
D_{n}=n ! \sum_{k=0}^{n} \frac{(-1)^{k}}{k !} \\
C_{n}=\frac{1}{n+1}\left(\begin{array}{c}
2 n \\
n
\end{array}\right) \\
E_{n}=n ! \cdot\left[t^{n}\right] y(t), \quad \text { where } y(t)=\tan (t)+\sec (t) \\
T_{n}=n^{n-2} .
\end{gathered}
$$

In all four cases, the corresponding formulas are equivalent by mathematical reasoning. Whether or not you accept (1.5)-(1.8) as formulas, it is their meaning that's important, not their form.

Finally, consider the following equations for the number of partitions $p(n)$, and primecounting function $\pi(n)$ :

$$
\begin{gathered}
p(n)=\left[t^{n}\right] \prod_{i=1}^{\infty} \frac{1}{1-t^{i}} \\
\pi(n)=\sum_{k=2}^{n}\left(\left\lfloor\frac{(k-1) !+1}{k}\right\rfloor-\left\lfloor\frac{(k-1) !}{k}\right\rfloor\right) .
\end{gathered}
$$

Equation (1.13) is due to Euler (1748), and had profound implications in number theory and combinatorics, initiating the whole area of partition theory (see e.g. [9]). Equation (1.14) follows easily from Wilson's theorem (see e.g. [40, §1.2.5]). Esthetic value aside, both equations are largely unhelpful for computing purposes and follow directly from definitions. Indeed, the former is equivalent to the standard counting algorithm (dynamic programming), while the latter is an iterated divisibility testing in disguise.

In summary, we see that the notion of "good formula" is neither syntactic nor semantic. One needs to make a choice depending on the application. 
1.2. Wilfian formulas. In his pioneering 1982 paper [174], Wilf proposed to judge a formula from the complexity point of view. He suggested two definitions of "good formulas" for computing an integer sequence $\left\{a_{n}\right\}$ :

(W1) There is an algorithm that computes $a_{n}$ in time poly $(n)$.

(W2) There is an algorithm that computes $a_{n}$ in time $o\left(a_{n}\right)$.

In the literature, such algorithms are called sometimes Wilfian formulas. Note that (W1) is aimed (but not restricted) to apply to sequences $\left\{a_{n}\right\}$ of at most exponential growth $a_{n}=$ $\exp O\left(n^{c}\right)$, while (W2) for $\left\{a_{n}\right\}$ of at most polynomial growth. See e.g. [66, 59] for more on growth of sequences.

Going over our list of examples we conclude that (1.1), (1.2), (1.4), (1.9), (1.10) and (1.12) are all transparently Wilfian of type (W1). Equations (1.3), (1.6), (1.7) and (1.11) are Wilfian of type (W1) in a less obvious but routine way (see below). Equations (1.3) and (1.5) do give rise to ad hoc poly $(n)$ algorithms, but care must be applied when dealing with irrational numbers. E.g., one must avoid circularity, such as when computing $n$-th prime $p_{n}$ by using the prime constant $\sum_{n} 1 / 2^{p_{n}}$, see e.g. [36, $\left.\$ 1.2 .5\right]$ and [158, A051006]. Finally, equation (1.8) is not Wilfian of type (W1), while (1.14) is not Wilfian of type (W2).

Let us add two more notions of a "good formula" in the same spirit, both of which are somewhat analogous but more useful than (W2):

(W3) There is an algorithm that computes $a_{n}$ in time poly $(\log n)$.

(W4) There is an algorithm that computes $a_{n}$ in time $n^{o(1)}$.

Now, for a combinatorial sequence $\left\{a_{n}\right\}$ one can ask if there is a Wilfian formula. In the original paper [174] an explicit example is given:

Conjecture 1.1 (Wilf). Let $a_{n}$ be the number of unlabeled graphs on $n$ vertices. Then $\left\{a_{n}\right\}$ has no Wilfian formula of type (W1).

See [158, A000088] for this sequence. Note that by the classical Erdös-Rényi result [55] (see also [13, §1.6]), we have $a_{n} \sim 2\left(\begin{array}{c}n \\ 2\end{array}\right) / n$ !, so the problem is not approximating $a_{n}$, but computing it exactly. For comparison, the sequence $\left\{c_{n}\right\}$ of the number of connected (labeled) graphs does have a Wilfian formula of type (W1):

$$
c_{n}=2^{\left(\begin{array}{c}
n \\
2
\end{array}\right)}-\frac{1}{n} \sum_{k=1}^{n-1} k\left(\begin{array}{l}
n \\
k
\end{array}\right) 2^{\left(\begin{array}{c}
n-k \\
2
\end{array}\right)} c_{k}
$$

(see [158, A001187] and [83, p. 7]).

The idea behind Conjecture 1.1 is that Pólya theory formulas (see e.g. 83]) are fundamentally not Wilfian. We should mention that we do not believe the conjecture in view of Babai's recent quasipolynomial time algorithm for GRAPH IsOMORPHISM [14]. While the connection is indirect, it is in fact conceivable that both problems can be solved in poly $(n)$ time.

Question 1.2. Let $\pi(n)$ denote the number of primes $\leq n$. Does $\{\pi(n)\}$ have a Wilfian formula of type (W4)?

Initially, Wilf asked about formula of type (W2) for $\{\pi(n)\}$, and such algorithm was given in [110]. Note that even the parity of $\pi(n)$ is hard to compute (cf. [165]), making unlikely a positive answer to the question above. 
1.3. Complexity setting and graph enumeration. Let $\mathcal{P}_{n}$ denote the set of certain combinatorial objects of size $n$. Formally, this means that one can decide if $X \in \mathcal{P}_{n}$ in time poly $(n)$. The problem of computing $a_{n}:=\left|\mathcal{P}_{n}\right|$ has the input $n$ of bit-length $O(\log n)$, much too small for the (usual) polynomial hierarchy. Instead, the exponential hierarchy is used: NEXP for existence of combinatorial objects which can be verified in time $n^{O(1)}$, and \#EXP for counting such objects 1$]$

For example, let $a_{n}=\left|\mathcal{P}_{n}\right|$ be the set of (labeled) planar 3-regular 3-connected graphs on $n$ vertices. Graphs in $\mathcal{P}_{n}$ are exactly graphs of simple 3-dimensional polytopes. Since testing each property can be done in $\operatorname{poly}(n)$ time, the decision problem is naturally in NEXP, and the counting problem is in \#EXP. In fact, the decision problem is trivially in $P$, since such graphs exist for all even $n \geq 4$ and don't exist for odd $n$. Furthermore, Tutte's formula for the number of rooted plane triangulations gives a simple product formula for $a_{n}$, and thus can be computed in poly $(n)$ time, see [168, Ch. 10].

On the one hand, counting the number of non-Hamiltonian graphs in $\mathcal{P}_{n}$ is not naturally in \#EXP, since testing non-Hamiltonicity is co-NP-complete in this case [64. On the other hand, the corresponding decision problem (the existence of such graphs) is again in $\mathrm{P}$ by Tutte's disproof of Tait's conjecture, see [168, Ch. 2].

Note that Graph Isomorphism is in P for trees, planar graphs and graphs of bounded degree, see e.g. [13, §6.2]. The discussion above suggests the following counterpart of Wilf's Conjecture 1.1

Conjecture 1.3. Let $a_{n}$ be the number of unlabeled plane triangulations with $n$ vertices, and let $b_{n}$ be the number of 3-connected planar graphs with $n$ vertices. Then $\left\{a_{n}\right\}$ and $\left\{b_{n}\right\}$ can be computed in $\operatorname{poly}(n)$ time.

We are very optimistic about this conjecture as for maps this is already known 63]. For triangulations there is some recent evidence in [93].

Theorem 1.4. Let $a_{n}$ be the number of unlabeled trees with $n$ vertices. Then $\left\{a_{n}\right\}$ can be computed in $\operatorname{poly}(n)$ time.

Proof. Denote by $b_{n}$ the number of unlabeled rooted trees with $n$ vertices. We have $b_{n} \leq C_{n-1}$ since the Catalan number $C_{n}$ is the number of plane trees with $n+1$ vertices, so $\log b_{n}=O(n)$. We also have:

$$
b_{n+1}=\frac{1}{n} \sum_{k=1}^{n} b_{n-k+1}\left[\sum_{d \mid k} d b_{d}\right],
$$

see e.g. [57, §5.6] and [158, A000081]. Thus, $\left\{b_{n}\right\}$ can be computed in poly $(n)$ time. On the other hand:

$$
a_{n}=b_{n}-\frac{1}{2} \sum_{k=1}^{n-1} b_{k} b_{n-k}+\left\{\begin{array}{cc}
\frac{1}{2} b_{n / 2}, & n \text { even } \\
0, & \text { otherwise }
\end{array}\right.
$$

see e.g. [83, §3.2] and [158, A000055]. This implies the result.

Let $a_{n}$ denote the number of 3-regular labeled graphs on $2 n$ vertices. The sequence $\left\{a_{n}\right\}$ can be computed in polynomial time via the following recurrence relation, see [76] and [158, A002829.

\footnotetext{
${ }^{1}$ To bring the problem into the polynomial hierarchy, the input $n$ should be given in unary.
} 


$$
\begin{aligned}
& 3(3 n-7)(3 n-4) \cdot a_{n}=9(n-1)(2 n-1)(3 n-7)\left(3 n^{2}-4 n+2\right) \cdot a_{n-1} \\
& \quad+(n-1)(2 n-3)(2 n-1)\left(108 n^{3}-441 n^{2}+501 n-104\right) \cdot a_{n-2} \\
& \quad+2(n-2)(n-1)(2 n-5)(2 n-3)(2 n-1)(3 n-1)\left(9 n^{2}-42 n+43\right) \cdot a_{n-3} \\
& \quad-2(n-3)(n-2)(n-1)(2 n-7)(2 n-5)(2 n-3)(2 n-1)(3 n-4)(3 n-1) \cdot a_{n-4}
\end{aligned}
$$

It was shown in [71] that similar polynomial recurrences exist for the number of $k$-regular labeled graphs, for every fixed $k \geq 1$.

Conjecture 1.5. Fix $k \geq 1$ and let $a_{n}$ be the number of unlabeled $k$-regular graphs with $n$ vertices. Then $\left\{a_{n}\right\}$ can be computed in $\operatorname{poly}(n)$ time.

For $k=1,2$ the problem is elementary, but for $k=3$ is related to enumeration of certain 2-groups (cf. [118]).

Consider now the problem of computing the number $f(m, n)$ of triangulations of an integer $[m \times n]$ grid (see Figure 1). This problem is a distant relative of Catalan numbers $C_{n}$ in (1.10) which Euler proved counts the number of triangulations of a convex $(n+2)$-gon (see [162]), and is one of the large family of triangulation problems (see 42]). Kaibel and Ziegler prove in 92 that $f(m, n)$ can be computed in $\operatorname{poly}(n)$ time for every fixed $m$, but report that their algorithm is expensive even for relatively small $m$ and $n$ (see [158, A082640]).

Question 1.6. Can $\{f(n, n)\}$ can be computed in poly $(n)$ time?
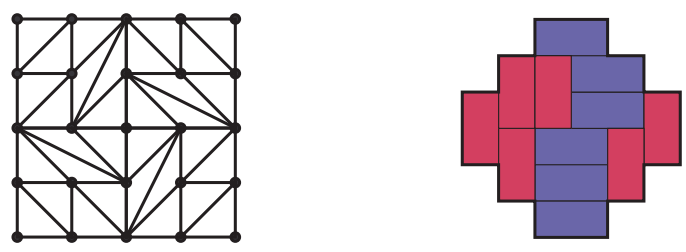

Figure 1. Grid triangulation of $[5 \times 5]$ and a domino tiling.

1.4. Computability setting and polyomino tilings. Let $a_{n}$ be the number of domino tilings on a $[2 n \times 2 n]$ square. The Kasteleyn and Temperley-Fisher classical determinant formula (1961) for the number of perfect matchings of planar graphs gives a poly $(n)$ time algorithm for computing $\left\{a_{n}\right\}$, see e.g. 96, 117]. This foundational result opens the door to potential generalizations, but, unfortunately, most of them turn out to be computationally hard.

First, one can ask about computing the number $b_{n}$ of 3 -dimensional domino tilings of a $[2 n \times 2 n \times 2 n]$ box. Or how about the seemingly simpler problem of counting the number $c_{n}$ of 3 -dimensional domino tilings of a "slim" $[2 \times n \times n]$ box? We don't know how to solve either problem, but both are likely to be difficult. The negative results include \#P-completeness of the counting problem for general and slim regions [141, 169], and topological obstacles, see [61 and [141, Prop. 8.1].

Now consider a fixed finite set $\mathbf{T}=\left\{\tau_{1}, \ldots, \tau_{k}\right\}$ of general polyomino tiles on a square grid: $\tau_{i} \subset \mathbb{Z}^{2}, 1 \leq i \leq k$. To tile a region $\Gamma \subset \mathbb{Z}^{2}$, one must cover it with copies of the tiles without overlap. These copies must be parallel translations of $\tau_{i}$ (rotations and reflections are not allowed). There exist NP-complete tileability problems and \#P-complete tiling counting 
problems even for a fixed set of a few small tiles. We refer to [134 for short survey of the area.

For a fixed $\mathbf{T}$, let $g(m, n)$ denote the number of tilings of $[m \times n]$ with $\mathbf{T}$. Is $g(m, n)$ computable in polynomial time? The following conjecture suggests otherwise.

Conjecture 1.7. There exists a finite set of tiles $\mathbf{T}$ such that counting the number of tilings of $[n \times n]$ with $\mathbf{T}$ is \#EXP-complete.

In fact, until we started writing this survey, we always believed this result to be known, only to realize that standard references such as [54] fall a bit short. Roughly, one needs to embed a \#EXP-complete language into a counting tilings problem of a rectangle. This is a classical idea (see e.g. [126, §5.3.4, §7.6.5]), which worked well for many related problems. For example, the Rectangular Tileability problem asks: given a finite set of tiles $\mathbf{T}$, do there exist integers $m$ and $n$, such that $\mathbf{T}$ tiles $[m \times n]$ ?

Theorem 1.8 (Yang [177]). The Rectangular Tileability problem is undecidable.

In the proof, Yang embeds the Halting Problem into Rectangular Tileability. So can one embed a NEXP-complete problem into tileability of an $[m \times n]$ rectangle? The answer is yes if $\mathbf{T}$ is allowed to be part of the input. In fact, even Levin's original 1973 paper introducing NP-completeness proposed this approach [113]. The following result should come as a surprise, perhaps.

Theorem 1.9 (Lam-Miller-Pak, see [111]). Given $\mathbf{T}$, the tileability of $[m \times n]$ can be decided in $O(\log m+\log n)$ time.

The proof is nonconstructive; it is based on Hilbert's Basis Theorem and the algebraic approach by F. W. Barnes. A combination of theorems 1.8 and 1.9 shows that the constant implied by the $O(\cdot)$ notation is not computable as a function of $\mathbf{T}$. Roughly, we do know that a linear-time algorithm exists, but given $\mathbf{T}$ it is undecidable to find it. Theorem 1.9 also explains why Conjecture 1.7 remains open - most counting results in the area use parsimonious reductions (think bijections between solutions of two problems), and in this case a different approach is required.

\section{Classes of Combinatorial sequences}

2.1. Algebraic and D-algebraic approach. Combinatorial sequences $\left\{a_{n}\right\}$ are traditionally classified depending on the algebraic properties of their GFs

$$
A(t)=\sum_{n=0}^{\infty} a_{n} t^{n} .
$$

We list here only four major classes:

Rational: $\quad A(t)=P(t) / Q(t)$, for some $P, Q \in \mathbb{Z}[t]$,

Algebraic: $\quad c_{0} A^{k}+c_{1} A^{k-1}+\ldots+c_{k}=0$, for some $k \in \mathbb{N}, c_{i} \in \mathbb{Z}[t]$,

D-finite: $\quad c_{0} A+c_{1} A^{\prime}+\ldots+c_{k} A^{(k)}=b$, for some $k \in \mathbb{N}, b, c_{i} \in \mathbb{Z}[t]$,

D-algebraic: $Q\left(t, A, A, \ldots, A^{(k)}\right)=0$, for some $k \in \mathbb{N}, Q \in \mathbb{Z}\left[t, x_{0}, x_{1}, \ldots, x_{k}\right]$.

Here we exclude the trivial equation $0=0$. Note that rational GFs are exactly those $\left\{a_{n}\right\}$ that satisfy a linear recurrence:

$$
c_{0} a_{n}=c_{1} a_{n-1}+\ldots+c_{k} a_{n-k}, \text { for some } k \in \mathbb{N}, c_{i} \in \mathbb{Z} .
$$


Such sequences $\left\{a_{n}\right\}$ are called $C$-recursive (or linearly recursive). For example, Fibonacci numbers satisfy (1.1) and have GF $\left(1-t-t^{2}\right)^{-1}$. Similarly, Catalan numbers have an algebraic GF by (1.6). D-finite GFs (also called holonomic) are exactly those $\left\{a_{n}\right\}$ that satisfy polynomial recurrence

$$
c_{0}(n) a_{n}=c_{1}(n) a_{n-1}+\ldots+c_{k}(n) a_{n-k}, \text { for some } k \in \mathbb{N}, c_{i} \in \mathbb{Z}[n] .
$$

Such sequences $\left\{a_{n}\right\}$ are called P-recursive. Examples include $\{n !\}$, derangement numbers $\left\{D_{n}\right\}$ by (1.9), the number of 3-regular graphs by (1.15), and the numbers $\left\{r_{n}\right\}$ of involutions in $S_{n}$, which satisfy $r_{n}=r_{n-1}+(n-1) r_{n-2}$, see [158, A000085]. Finally, D-algebraic GFs (also called $A D E$ and hyperalgebraic) include Euler numbers by equation (1.7) and the number of partitions $p(n)$, see below.

Theorem 2.1 (see e.g. [160, Ch. 6]).

\section{Rational $\subset$ Algebraic $\subset D$-finite $\subset$ D-algebraic.}

Here only the inclusion Algebraic $\subset D$-finite is nontrivial. The following easy observation explains the connection to the subject (we omit the proof).

Proposition 2.2. Sequences with D-algebraic GFs have Wilfian formulas of type (W1).

In other words, if one wants to show that a sequence does not have a Wilfian formula, then proving that it is D-transcendental, i.e. non-D-algebraic, is a good start 2 Unfortunately, even proving that a sequence is non-P-recursive is often challenging (see below).

Example 2.3 (Bell numbers). Let $B_{n}$ denote the number of set partitions of $\{1, \ldots, n\}$, see [160] and [158, A000110]. Let

$$
y(t)=\sum_{n=0}^{\infty} \frac{B_{n} t^{n}}{n !}, \quad z(t)=\sum_{n=0}^{\infty} B_{n} t^{n},
$$

be the exponential and ordinary GFs of Bell numbers, respectively. On the one hand, we have:

$$
y(t)=e^{e^{t}-1}, \quad y^{\prime \prime} y-\left(y^{\prime}\right)^{2}-y^{\prime} y=0 .
$$

Thus, $y(t)$ is D-algebraic, and the proposition implies that $\left\{B_{n}\right\}$ can be computed in poly $(n)$ time. On the other hand, $z(t)$ is D-transcendental by Klazar's theorem [100].

This also implies that $y(t)$ is not D-finite. Indeed, observe by definition, that if a sequence $\left\{a_{n}\right\}$ is P-recursive, then so is $\left\{n ! a_{n}\right\}$, which implies the result by taking $a_{n}=B_{n} / n$ ! (cf. [114). Of course, there is a more direct way to prove that $y(t)$ is not $D$-finite by repeated differentiation or via the asymptotics, see below. This suggests the following advanced generalization of Klazar's theorem.

Open Problem 2.4 (Pak-Yeliussizov). Suppose $\left\{a_{n} / n !\right\}$ is D-algebraic but not P-recursive. Does this imply that $\left\{a_{n}\right\}$ is D-transcendental?

Before we proceed to more combinatorial examples, let us mention that D-transcendental GFs are the subject of Differential Galois Theory, which goes back to Liouville, Lie, Picard and Vessiot in the 19th century (see e.g. 151]), and continues to be developed [149]. Some natural GFs are known to be D-transcendental, e.g. $\Gamma(z), \zeta(z)$, etc., but there are too few methods to prove this in most cases of interest. Here are some of our favorite open problems along these lines, unapproachable with existing tools.

\footnotetext{
${ }^{2}$ To simplify exposition and for the lack of better terminology, here and in the future we refer to sequences by the properties of their GFs.
} 
Conjecture 2.5. $\sum_{n \geq 1} p_{n} t^{n}$ and $\sum_{n \geq 1} \pi(n) t^{n}$ are D-transcendental.

Here $p_{n}$ is the $n$-th prime, and $\pi(n)$ is the number of primes $\leq n$, as above. Both GFs are known to be non-D-finite, as shown by Flajolet, Gerhold and Salvy in [58] by asymptotic arguments. The authors quip: "Almost anything is non-holonomic unless it is holonomic by design". Well, maybe so. But the same applies for D-transcendence where the gap between what we believe and what we can prove is much wider. The reader should think of such open problems as the irrationality of $e+\pi$ and $\zeta(5)$, and imagine a similar phenomenon in this case (cf. [102]).

Conjecture 2.6. $\sum_{n \geq 0} t^{n^{3}}$ is D-transcendental.

This problem should be compared with Jacobi's 1848 theorem that the theta function $\sum_{n} t^{n^{2}}$ is D-algebraic [90. To understand the difference, the conjecture is saying that there are no good formulas governing the number of ways to write $n$ as a sum of $k$ cubes, for any $k$, the kind of formulas that exist for sums of two, four and six squares, see [84, $\S \mathrm{XX}]$.

2.2. Combinatorial and asymptotic tools. The following is a simple combinatorial criterion for non-P-recursiveness.

Theorem 2.7 ([66]). Let $\left\{a_{n}\right\}$ be a P-recursive integer sequence. Consider the infinite binary word $\mathbf{w}=w_{1} w_{2} \cdots$ defined by $w_{n}=a_{n} \bmod 2$. Then there exists a finite binary word $v$ that is not a subword of $w$.

Here by a subword we mean a consecutive subsequence of letters. For example, the infinite binary word for the sequence of Fibonacci numbers $\left\{F_{n} \bmod 2\right\}$ do not contain (111), Catalan numbers $\left\{C_{n} \bmod 2\right\}$ and derangement numbers $\left\{D_{n} \bmod 2\right\}$ do not contain (11), etc. In a different direction, this implies that the binary Champernowne sequence (all natural numbers in binary, concatenated)

$$
0,1,0,0,0,1,1,0,1,1,0,0,0,0,0,1,0,1,0,0,1,1, \ldots
$$

is not P-recursive, see [158, A076478].

Unfortunately, for many natural non-P-recursive sequences the assumption in the theorem is much too strong. For example, for the parity of partition numbers $\{p(n) \bmod 2\}$, and for the odd primes modulo $4,\left\{\left(p_{n}-1\right) / 2 \bmod 2\right\}$, it is an open problem whether all binary subwords appear (bet on yes).

The following result is the best tool we have for proving that a combinatorial sequence is not P-recursive. Note that deriving such asymptotics can be very difficult; we refer to [59, 145] for recent comprehensive monographs on the subject.

Theorem 2.8. Let $\left\{a_{n}\right\}$ be a P-recursive sequence, s.t. $a_{n} \in \mathbb{Q}, C_{1}^{n}<a_{n}<C_{2}^{n}$ for some $C_{2}>C_{1}>0$ and all $n \geq 1$. Then

$$
a_{n} \sim \sum_{i=1}^{m} K_{i} \lambda_{i}^{n} n^{\alpha_{i}}(\log n)^{\beta_{i}},
$$

where $K_{i} \in \mathbb{R}_{+}, \lambda_{i} \in \overline{\mathbb{Q}}, \alpha_{i} \in \mathbb{Q}$, and $\beta_{i} \in \mathbb{N}$.

The theorem is a combination of several known results [66]. Briefly, the generating series $\mathcal{A}(t)$ is a $G$-function in a sense of Siegel (1929), which by the works of André, Bombieri, Chudnovsky, Dwork and Katz, must satisfy an ODE which has only regular singular points 
and rational exponents. We then apply the Birkhoff-Trjitzinsky claim/theorem, which in the regular case has a complete and self-contained proof in [59] (see Theorem VII.10 and subsequent comments).

Example 2.9 (Euler numbers $E_{n}$ ). Recall that

$$
E_{n} \sim \frac{4}{\pi}\left(\frac{2}{\pi}\right)^{n} n !
$$

(see e.g. [59, p. 7]). Then $\left\{E_{n}\right\}$ is not P-recursive, since otherwise $E_{n} / n ! \sim K \lambda^{N}$ with a transcendental base of exponent $\lambda=(2 / \pi) \notin \overline{\mathbb{Q}}$. Note that Euler numbers can be computed in time $O\left(n^{\frac{4}{3}+\epsilon}\right)$ 85].

Example 2.10 $\left(n\right.$-th prime $\left.p_{n}\right)$. Following [58, recall that $p_{n}=n \log n+n \log \log n+O(n)$. Observe that the harmonic number $h_{n}$ is P-recursive by definition:

$$
h_{n}=h_{n-1}+\frac{1}{n}=1+\frac{1}{2}+\ldots+\frac{1}{n}=\log n+O(1) .
$$

Then $\left\{p_{n}\right\}$ is not P-recursive, since otherwise so is

$$
p_{n}-n h_{n}=n \log \log n+O(n),
$$

which is impossible by Theorem 2.8 ,

2.3. Lattice walks. Let $\Gamma=(V, E)$ be a graph and let $v_{0}, v_{1} \in V$ be two fixed vertices. Let $a_{n}$ be the number of walks $v_{0} \rightarrow v_{1}$ in $\Gamma$ of length $n$. This is a good model which leads to many interesting sequences. For example, Fibonacci number $F_{n}$ is the number of walks $1 \rightarrow 1$ of length $n$ in the graph on $\{1,2\}$, with edges $(1,1),(1,2)$ and $(2,1)$.

For general finite graphs we get C-recursive sequences $\left\{a_{n}\right\}$ with rational GFs. For the usual walks $0 \rightarrow 0$ on $\mathbb{N}$ we get Catalan numbers $a_{2 n}=C_{n}$ as in (1.10), while for \pm 1 walks in $\mathbb{Z}$ we get $a_{2 n}=\left(\begin{array}{c}2 n \\ n\end{array}\right)$, both algebraic sequences. Similarly, for $(0, \pm 1),( \pm 1,0)$ walks in $\mathbb{Z}^{2}$, we get $a_{2 n}=\left(\begin{array}{c}2 n \\ n\end{array}\right)^{2}$, which is P-recursive but not algebraic [62]. In higher dimensions or for more complicated graphs, there is no such neat formula.

Theorem 2.11. Let $S \subset \mathbb{Z}^{d}$ be a fixed finite set of steps, and let $a_{n}$ be the number of walks $O \rightarrow O$ in $\mathbb{Z}^{d}$ of length $n$, with steps in $S$. Then $\left\{a_{n}\right\}$ is P-recursive.

This result is classical and follows easily from [160, §6.3]. It suggests that to obtain more interesting sequences one needs to look elsewhere. Notably, one can consider natural lattice walks on some portion of $\mathbb{Z}^{d}$. There is a tremendous number of results in the literature, remarkable both in scope and beauty.

In recent years, M. Bousquet-Mélou and her coauthors initiated a broad study of the subject, and now have classified all walks in the first quadrant which start and end at the origin $O$, and have a fixed set $S$ of steps with both coordinates in $\{0, \pm 1\}$. There are in principle $2^{8}-1=255$ such walks, but some of them are trivial and some are the same up to symmetries. After the classification was completed, some resulting sequences were proved algebraic (say, Kreweras walks and Gessel walks); very surprisingly so, some are D-finite (not a surprise given Theorem 2.11), some are D-algebraic (this required development of new tools), and some are D-transcendental (it is amazing that this can be done at all). 
Example 2.12 (Case 16). Let $S=\{(1,1),(-1,-1),(-1,0),(0,-1)\}$, and let $a_{n}$ be the number of walks $O \rightarrow O$ in the first quadrant of length $n$, with steps in $S$, see [158, A151353]. It was shown in [23, Case 16] that

$$
a_{n} \sim K \lambda^{n} n^{\alpha},
$$

where $\lambda \approx 3.799605$ is a root of $x^{4}+x^{3}-8 x^{2}-36 x-11=0$, and $\alpha \approx-2.318862$ satisfies $c=-\cos (\pi / \alpha)$, and $c$ is a root of

$$
y^{4}-\frac{9}{2} y^{3}+\frac{27}{4} y^{2}-\frac{35}{8} y+\frac{17}{16}=0 .
$$

Since $\alpha \notin \mathbb{Q}$, Theorem 2.8 implies that $\left\{a_{n}\right\}$ is not P-recursive.

We refer to [25, 26] for a comprehensive overview of the background and early stages of this far-reaching project, and to [15, 22] for some recent developments which are gateways to references. Finally, let us mention a remarkable recent development [49], which proves D-transcendence for many families of lattice walks. Let us single out just one of the many results in that paper:

Theorem 2.13 ([49, Thm. 5.8]). Sequence $\left\{a_{n}\right\}$ defined in Example 2.12 is D-transcendental.

In conclusion, let us mention that $\left\{a_{n}\right\}$ can be computed in polynomial time straight from definition using dynamic programming, since the number of points reachable after $n$ steps is poly $(n)$. This leads us to consider walks with constraints or graphs of superpolynomial growth.

Conjecture 2.14 (cf. [179]). Let $a_{n}$ denotes the number of self-avoiding walks $O \rightarrow O$ in $\mathbb{Z}^{2}$ of length $n$. Then the sequence $\left\{a_{n}\right\}$ has no Wilfian formula of type (W1).

We refer to [79] for an extensive investigation of self-avoiding walks and its relatives, and a review of the literature.

2.4. Walks on Cayley graphs. Let $G=\langle S\rangle$ be a finitely generated group $G$ with a generating set $S$. Let $a_{n}=a_{n}(G, S)$ be the number of words in $S$ of length $n$ equal to 1; equivalently, the number of walks $1 \rightarrow 1$ of length $n$, in the Cayley graph $\Gamma=\Gamma(G, S)$. In this case $\left\{a_{n}\right\}$ is called the cogrowth sequence and its GF $A(t)$ the cogrowth series. They were introduced by Pólya in 1921 in the probabilistic context of random walks on graphs, and by Kesten in the context of amenability [97.

The cogrowth sequence $\left\{a_{n}\right\}$ is C-recursive if only if $G$ is finite [108. It is algebraic for the infinite dihedral group [88], for the free group [80] and for free products of finite groups [109], all with standard generators. The cogrowth sequence is P-recursive for many abelian groups [88, and for the Baumslag-Solitar groups $G=\mathrm{BS}(k, k)$ in the standard presentation $\mathrm{BS}(k, \ell)=\left\langle x, y \mid x^{k} y=y x^{\ell}\right\rangle$, see [53].

Theorem 2.15 ([66]). The sequence $\left\{a_{n}(G, S)\right\}$ is not P-recursive for all symmetric $S=$ $S^{-1}$, and the following classes of groups $G$ :

(1) virtually solvable groups of exponential growth with finite Prüfer rank;

(2) amenable linear groups of superpolynomial growth;

(3) groups of weakly exponential growth

$$
A e^{n^{\alpha}}<\gamma_{G, S}(n)<B e^{n^{\beta}}
$$

where $A, B>0$, and $0<\alpha, \beta<1$;

(4) the Baumslag-Solitar groups $\mathrm{BS}(k, 1)$, where $k \geq 2$;

(5) the lamplighter groups $L(d, H)=H \imath \mathbb{Z}^{d}$, where $H$ is a finite abelian group and $d \geq 1$. 
Since $G \simeq \mathbb{Z} \ltimes \mathbb{Z}^{2}$ with a free action of $\mathbb{Z}$, is linear of exponential growth, by (2) we obtain a solution to the question originally asked by Kontsevich, see [161].

Corollary 2.16 ([66]). There is a linear group $G$ and a symmetric generating set $S$, s.t. the sequence $\left\{a_{n}(G, S)\right\}$ is not P-recursive.

The proof in 66 is a combination of many results by different authors. For example, for $G=\operatorname{BS}(k, 1), k \geq 2$, and every symmetric $\langle S\rangle=G$, there exist $C_{1}, C_{2}>0$ that depend on $S$, s.t.

$$
|S|^{n} e^{-C_{1} \sqrt[3]{n}} \leq a_{n}(G, S) \leq|S|^{n} e^{-C_{2} \sqrt[3]{n}},
$$

see [176, §15.C]. The result now follows from Theorem 2.8 .

It may seem from Theorem 2.15 that the properties of $\left\{a_{n}(G, S)\right\}$ depend only on $G$, but that is false. In fact, for $G=F_{k} \times F_{\ell}$ there are generating sets with both P-recursive and non-P-recursive sequences; the negative result in this case is given is proved in [66] by using Theorem 2.7. For groups in Theorem 2.15, the result is a byproduct of probabilistic tools used in establishing the asymptotics such as (2.1). In fact, the probabilities of return of the random walk $a_{n}(G, S) /|S|^{n}$ always have the same growth under quasi-isometry, see e.g. 176] 3

It is unlikely that any of the sequences in the theorem are D-algebraic, but we really have no idea nor any tools to establish such a result other than by a direct calculation. An exact asymptotic result is known for a particular walk on the lamplighter group is given in [150]. Let $G=L\left(1, \mathbb{Z}_{2}\right)=\mathbb{Z}_{2}$ 々 $\mathbb{Z}$, with a symmetric generating (multi-)set $S=S_{1} S_{2} S_{1}$, where $S_{1}=\{(1,0), e\}$ and $S_{2}=\{(0, \pm 1)\}$. In other words, each generator is a sequence of moves: turn the lamp on or off, make a step left or right, then turn the lamp on or off at the new location, all with probability $1 / 2$. Then:

$$
a_{n}(G, S)=K n^{1 / 6} e^{-C \sqrt[3]{n}}, \quad \text { where } K=\frac{2^{2 / 3} \pi^{5 / 6}}{3^{1 / 2}(\log 2)^{2 / 3}}, \quad C=3 \cdot 2^{1 / 3}(\pi \log 2)^{2 / 3} .
$$

It would be interesting to see if this sequence is D-algebraic.

In a forthcoming paper [67 we construct an explicit but highly artificial non-symmetric set $S \subset F_{k} \times F_{\ell}$ with D-transcendental cogrowth sequence. In 95, we use the tools in 94 to prove that groups have an uncountable set of spectral radii

$$
\rho(G, S):=\lim _{n \rightarrow \infty} a_{n}(G, S)^{1 / n} .
$$

Since the set of D-algebraic sequence is countable, this implies the existence of D-transcendental Cayley graphs with symmetric $S$, but such a proof is nonconstructive.

Open Problem 2.17. Find an explicit construction of $\Gamma(G, S)$ when $S$ is symmetric, and $\left\{a_{n}(G, S)\right\}$ is D-transcendental.

The sequences $\left\{a_{n}\right\}$ have been computed in very few special cases. For example, for $\operatorname{PSL}(2, \mathbb{Z})=\mathbb{Z}_{2} * \mathbb{Z}_{3}$ with the natural symmetric generating set, the cogrowth series $A(t)$ is computed in [109]:

$$
\begin{aligned}
& A(t)=\frac{(1+t)\left(-t+t^{2}-8 t^{3}+3 t^{4}-9 t^{5}+\left(2-t+6 t^{2}\right) \sqrt{\mathcal{R}(t)}\right)}{2(1-3 t)\left(1+3 t^{2}\right)\left(1+3 t+3 t^{2}\right)\left(1-t+3 t^{2}\right)}, \\
& \text { where } \mathcal{R}(t)=1-2 t+t^{2}-6 t^{3}-8 t^{4}-18 t^{5}+9 t^{6}-54 t^{7}+81 t^{8} .
\end{aligned}
$$

\footnotetext{
${ }^{3}$ While the leading term in the asymptotics remains the same, lower order terms can change for different $S$, see [176, §17.B].
} 
There are more questions than answers here. For example, can cogrowth sequence be computed for nilpotent groups?

Before we conclude, let us note that everywhere above we are implicitly assuming that $G$ either has a faithful rational representation, e.g. $G=\mathrm{BS}(k, 1)$ as in (4) above, or more generally has the word problem solvable in polynomial time (cf. [115]). The examples include the Grigorchuk group $\mathbb{G}$, which is an example of 3, see [78] and the lamplighter groups $L(d, H)$ as in (5). Note that in general the word problem can be superpolynomial or even unsolvable, see e.g. [123], in which case $\left\{a_{n}\right\}$ is no longer a combinatorial sequence.

2.5. Partitions. Let $p(n)$ be the number of integer partitions of $n$, as in (1.13). We have the Hardy-Ramanujan formula:

$$
p(n) \sim \frac{1}{4 n \sqrt{3}} e^{\pi \sqrt{\frac{2 n}{3}}} \text { as } n \rightarrow \infty .
$$

(see e.g. [59, VIII.6]). Theorem 2.8 implies that $\{p(n)\}$ is not P-recursive. On the other hand, it is known that

$$
F(t):=\sum_{n=0}^{\infty} p(n) t^{n}=\prod_{i=1}^{\infty} \frac{1}{1-t^{i}}
$$

satisfies the following ADE[

$$
\begin{aligned}
4 F^{3} F^{\prime \prime} & +5 t F^{3} F^{\prime \prime \prime}+t^{2} F^{3} F^{(4)}-16 F^{2}\left(F^{\prime}\right)^{2}-15 t F^{2} F^{\prime} F^{\prime \prime}-39 t^{2} F^{2}\left(F^{\prime \prime}\right)^{2} \\
& +20 t^{2} F^{2} F^{\prime} F^{\prime \prime \prime}+10 t F\left(F^{\prime}\right)^{3}+12 t^{2} F\left(F^{\prime}\right)^{2} F^{\prime \prime}+6 t^{2}\left(F^{\prime}\right)^{4}=0 .
\end{aligned}
$$

(cf. [122, 178]). A quantitative version of Proposition 2.2 then implies that $p(n)$ can be computed in time $O\left(n^{4.5+\epsilon}\right)$, for all $\epsilon>0$. For comparison, the dynamic programming takes $O\left(n^{2.5}\right)$ time, where $O(\sqrt{n})$ comes as the cost of addition. Similarly, Euler's recurrence famously used by MacMahon (1915) to compute $p(200)$, gives an $O\left(n^{2}\right)$ algorithm:

$$
p(n)=p(n-1)+p(n-2)-p(n-5)-p(n-7)+p(n-12)+p(n-15)-\ldots
$$

(cf. 32] ). Finally, there is nearly optimal $O\left(\sqrt{n}(\log n)^{4+\epsilon}\right)$ time algorithm given in [91. It is based on the Hardy-Ramanujan-Rademacher sharp asymptotic formula, which extends (2.2) to $o(1)$ additive error.

Now, for a subset $\mathcal{A} \subseteq\{1,2, \ldots\}$, let $p_{\mathcal{A}}(n)$ denote the number of partitions of $n$ into parts in $\mathcal{A}$. The dynamic programming algorithm is easy to generalize to every $\left\{p_{\mathcal{A}}(n)\right\}$ where the membership $a \in ? \mathcal{A}$ can be decided in poly $(\log a)$ time, giving a Wilfian formula of type (W1). This is polynomially optimal for partitions into primes [158, A000607] or squares [158, A001156], but not for sparse sequences.

Proposition 2.18. Let $\mathcal{A}=\left\{a_{1}, a_{2}, \ldots\right\}$, such that $a_{k} \geq c^{k}$, for some $c>1$ and all $k \geq 1$. Then $p_{\mathcal{A}}(n)=n^{O(\log n)}$.

Thus, $p_{\mathcal{A}}(n)$ as in the proposition could in principle have a Wilfian formula of type (W3). Notable examples include the number $q(n)$ of binary partitions (partitions of $n$ into powers of 2), see [158, A000123, partitions into Fibonacci numbers [158, A003107], and s-partitions defined as partitions into $\left\{1,3,7, \ldots, 2^{k}-1, \ldots\right\}$ [158, A000929].

Theorem 2.19 ([142]). Let $\mathcal{A}=\left\{a_{1}, a_{2}, \ldots\right\}$, and suppose $a_{k} / a_{k-1}$ is an integer $\geq 2$, for all $k>1$. Suppose also that membership $x \in \mathcal{A}$ can be decided in poly $(\log x)$ time. Then $\left\{p_{\mathcal{A}}(n)\right\}$ can be computed in time poly $(\log n)$.

\footnotetext{
${ }^{4}$ This equation was found by Martin Rubey, see https://tinyurl.com/y7ewapjc.
} 
This covers binary partitions, partitions into factorials [158, A064986, etc.

We conjecture that partitions into Fibonacci numbers and s-partitions also have Wilfian formulas of type (W3). Cf. 152 for an algorithm for partitions into distinct Fibonacci numbers. Note also that membership can be tested in polynomial time: $N$ is a Fibonacci number if and only if $5 N^{2}+4$ or $5 N^{2}-4$ is a perfect square [70].

Other partition sequences $\left\{p_{\mathcal{A}}(n)\right\}$ with $\mathcal{A}$ as in Proposition 2.18, could prove less tractable. These include partitions into Catalan numbers [158, A033552] and partitions into partition numbers [158, A007279.

We should mention that connection between algebraic properties of GFs and complexity goes only one way:

Theorem 2.20. The sequence $\{q(n)\}$ of the number of binary partitions is D-transcendental.

This follows from the Mahler equation

$$
Q(t)-t Q(t)-Q\left(t^{2}\right)=0, \quad \text { where } \quad Q(t)=\sum_{n=0}^{\infty} q(n) t^{n},
$$

see e.g. [48. We conjecture that $\left\{a_{n}\right\}$ and $\left\{b_{n}\right\}$ from Conjecture 1.3 satisfy similar functional equations, and are also D-transcendental.

2.6. Pattern avoidance. Let $\sigma \in S_{n}$ and $\omega \in S_{k}$. The permutation $\sigma$ is said to contain the pattern $\omega$ if there is a subset $X \subseteq\{1, \ldots, n\},|X|=k$, such that $\left.\sigma\right|_{X}$ has the same relative order as $\omega$. Otherwise, $\sigma$ is said to avoid $\omega$.

Fix a set of patterns $\mathcal{F} \subset S_{k}$. Let $\mathrm{A}_{n}(\mathcal{F})$ denote the number of permutations $\sigma \in S_{n}$ avoiding all patterns $\omega \in \mathcal{F}$. The sequence $\left\{\mathrm{A}_{n}(\mathcal{F})\right\}$ is the fundamental object of study in the area of pattern avoidance, extensively analyzed from analytic, asymptotic and combinatorial points of view.

The subject was initiated by MacMahon (1915) and Knuth (1973), who showed that $\mathrm{A}_{n}(123)=\mathrm{A}_{n}(213)=C_{n}$, the $n$-th Catalan number (1.10). The Erdös-Szekeres theorem (1935) on longest increasing and decreasing subsequences in a permutation can also be phrased in this language: $\mathrm{A}_{n}(12 \cdots k, \ell \cdots 21)=0$, for all $n>(k-1)(\ell-1)$.

To give a flavor of subsequent developments, let us mention a few more of our most favorite results. Simion-Schmidt (1985) proved $\mathrm{A}_{n}(123,132,213)=F_{n+1}$, the Fibonacci numbers. Similarly, Shapiro-Stephens (1991) proved $\mathrm{A}_{n}(2413,3142)=S(n)$, the Schröder numbers [158, A006318. The celebrated Marcus-Tardos theorem [121] states that $\left\{\mathrm{A}_{n}(\omega)\right\}$ is at most exponential, for all $\omega \in S_{k}$, with a large base of exponent for random $\omega \in S_{k}$ [60]. We refer to [99, 101, 170] for many results on the subject, history and background.

The Noonan-Zeilberger conjecture [130, first posed as a question by Gessel [71, states that the sequence $\left\{\mathrm{A}_{n}(\mathcal{F})\right\}$ is P-recursive for all $\mathcal{F} \subset S_{k}$. It was recently disproved:

Theorem 2.21 (65]). There is $\mathcal{F} \subset S_{80},|\mathcal{F}|<30,000$, such that $\left\{\mathrm{A}_{n}(\mathcal{F})\right\}$ is not $P$ recursive.

We extend this result in a forthcoming paper [67, where we construct a D-transcendent sequence $\left\{\mathrm{A}_{n}(\mathcal{F})\right\}$, for some $\mathcal{F} \subset S_{80}$. Both proofs involve embedding of Turing machines into the problem modulo 2. We also prove the following result on complexity of counting pattern-avoiding permutations, our only result forbidding Wilfian formulas:

Theorem $2.22([65])$. If EXP $\neq \oplus \mathrm{EXP}$, then $\mathrm{A}_{n}(\mathcal{F})$ mod 2 cannot be computed in poly $(n)$ time. 
Here $\oplus$ EXP is the class of counting modulo 2 problems of combinatorial objects in NEXP. In other words, computing parity of the number of pattern-avoiding permutations is likely hard. We conjecture that $\mathrm{A}_{n}(\mathcal{F})$ is \#EXP-complete, but we are not very close to proving this.

Theorem 2.23 ([65]). The problem whether $\mathrm{A}_{n}(\mathcal{F})=\mathrm{A}_{n}\left(\mathcal{F}^{\prime}\right)$ mod 2 for all $n$, is undecidable.

The theorem implies that in some cases even a large amount of computational evidence in pattern avoidance is misleading. For example, there exists two sets of patterns $\mathcal{F}, \mathcal{F}^{\prime} \in S_{k}$, so that the first time they have different parity is for $n>$ tower of $2 \mathrm{~s}$ of height $2^{k}$.

Finally, let us mention an ongoing effort to find a small set of patterns $\mathcal{F}$, so that $\left\{\mathrm{A}_{n}(\mathcal{F})\right\}$ is not P-recursive. Is one permutation enough? It is known that $\left\{\mathrm{A}_{n}(1342)\right\}$ is algebraic [20], while $\left\{\mathrm{A}_{n}(1234)\right\}$ is $\mathrm{P}$-recursive [71. One of the most challenging problems is to analyze $\left\{\mathrm{A}_{n}(1324)\right\}$, the only 4-pattern remaining. The asymptotics obtained experimentally in 38] based on the values for $n \leq 50$, suggests:

$$
\mathrm{A}_{n}(1324) \sim B \lambda^{n} \mu^{\sqrt{n}} n^{\alpha},
$$

where $\lambda=11.600 \pm 0.003, \mu=0.0400 \pm 0.0005, \alpha=-1.1 \pm 0.1$. By Theorem 2.8, this is a convincing evidence against $\left\{\mathrm{A}_{n}(1324)\right\}$ being P-recursive. While proving such a result remains out of reach, the following problem could be easier.

Open Problem 2.24. Can $\left\{\mathrm{A}_{n}(1324)\right\}$ be computed in poly $(n)$ time伺 More generally, can one find a single permutation $\pi$ such that $\left\{\mathrm{A}_{n}(\pi)\right\}$ cannot be computed in poly $(n)$ time? Is the computation of $\left\{\mathrm{A}_{n}(\pi)\right\}$ easier or harder for random permutations $\pi \in S_{k}$ ?

In the opposite direction, let us mention a sequence $\left\{\mathrm{A}_{n}(4123,4231,4312)\right\}$ which does have a Wilfian formula of type (W1), with an extremely strong computational evidence for being D-transcendental [6].

\section{BiJections}

3.1. Counting and sampling via bijections. There is an ocean of bijections between various combinatorial objects. They have a variety of uses: to establish a theorem, to obtain refined counting, to simplify the proof, to make the proof amenable for generalizations, etc. Last but not least, an especially beautiful bijection is often viewed as a piece of art, an achievement in its own right, a result to be taught and admired.

From the point of view of this survey, bijections $\varphi: \mathcal{A}_{n} \rightarrow \mathcal{B}_{n}$ are simply algorithms which require complexity analysis. There are two standard applications of such bijections. First, their existence allows us to reduce counting of $\left\{\left|\mathcal{A}_{n}\right|\right\}$ to counting of $\left\{\left|\mathcal{B}_{n}\right|\right\}$. For example, the classical Prüfer algorithm allows counting of spanning trees in $K_{n}$, reducing it to Cayley's formula (1.12).

Second and more recent application is to random sampling of combinatorial objects. Oftentimes, one of the sets has a much simpler structure which allows (nearly) uniform sampling. To compare the resulting algorithm with other competing approaches one then needs a worst case or average-case analysis of the complexity of the bijection.

Of course, most bijections in the literature are so straightforward that their analysis is elementary, think of the Prüfer algorithm or the classical "plane trees into binary trees" bijection [41]. But this is also what makes them efficient. For example, the bijections for

\footnotetext{
${ }^{5}$ In 2005, Doron Zeilberger expressed doubts that $\mathrm{A}_{1000}(1324)$ could be computed even by Hashem. This sentiment has been roundly criticized on both mathematical and theological grounds (see [163]).
} 
planar maps are amazing in their elegance, and have some important applications to statistical physics; we refer to [156] for an extensive recent survey and numerous references.

Finally, we should mention a number of perfect sampling algorithms, some of which in the right light can also be viewed as bijections. These include most notably general techniques such as Boltzmann samplers [51. (see also [12, 21), and coupling from the past 112. Note also two beautiful ad hoc algorithms: Wilson's LERW [175] and the Aldous-Broder algorithm for sampling uniform spanning trees in a graph (both of which are highly nontrivial already for $K_{n}$ ), see e.g. [112].

3.2. Partition bijections. This is a large subject in its own right, with many results and open problems. For example, the Bressoud-Zeilberger involution [30] proves Euler's recurrence (2.4). At the same time, the equation implied by the ADE recurrence (2.3) does not yet have a combinatorial proof, and looking for such a proof would not be advisable. We refer to [137] for an extensive survey.

Let $q(n)$ denote the number of concave partitions defined by $\lambda_{i}-\lambda_{i+1} \geq \lambda_{i+1}-\lambda_{i+2}$ for all $i$. Then $\{q(n)\}$ can be computed in poly $(n)$ time. To see this, recall Corteel's bijection between convex partitions and partitions into triangular numbers [158, A007294]. We then have:

$$
\sum_{n=1}^{\infty} q(n) t^{n}=\prod_{k=2}^{\infty} \frac{1}{1-t^{\left(\begin{array}{c}
k \\
2
\end{array}\right)}}
$$

see [34. This bijection can be described as a linear transformation that can be computed in polynomial time [39, 135]. More importantly, the bijections allow random sampling of concave partitions, leading to their limit shape [34, 44].

On the opposite extreme, there is a similar Hickerson bijection between s-partitions and partitions with $\lambda_{i} \geq 2 \lambda_{i+1}$ for all $i \geq 1$, see [34, 135]. Thus, both sets are equally hard to count, but somehow this makes the problem more interesting.

The Garsia-Milne celebrated involution principle [69] combines the Schur and Sylvester bijections in an iterative manner, giving a rather complicated bijective proof of the RogersRamanujan identity:

$$
1+\sum_{k=1}^{\infty} \frac{t^{k^{2}}}{(1-t)\left(1-t^{2}\right) \cdots\left(1-t^{k}\right)}=\prod_{i=0}^{\infty} \frac{1}{\left(1-t^{5 i+1}\right)\left(1-t^{5 i+4}\right)} .
$$

To be precise, they constructed a bijection $\Psi_{n}: \mathcal{P}_{n} \rightarrow \mathcal{Q}_{n}$, where $\mathcal{P}$ is the set of partitions into parts $\lambda_{i} \geq \lambda_{i+1}+2$, and $\mathcal{Q}$ is the set of partitions into parts $\pm 1 \bmod 5$. In [137, §8.4.5] we conjecture that $\Psi_{n}$ requires $\exp n^{\Omega(1)}$ iterations in the worst case. Partial evidence in favor of this conjecture is our analysis of O'Hara's bijection in [103], with an $\exp \Omega(\sqrt[3]{n})$ worstcase lower bound. On the other hand, the iterative proof in [24] for (3.1) requires only $O(n)$ iterations.

3.3. Plane partitions and Young tableaux. Denote by $p p(n)$ the number of plane (also called solid) partitions. MacMahon famously proved in 1912 that

$$
\sum_{n=0}^{\infty} p p(n) t^{n}=\prod_{k=1}^{\infty} \frac{1}{\left(1-t^{k}\right)^{k}},
$$

which gives a poly $(n)$ time algorithm for computing $s p(n)$. This identity follows from a variation on the classical Hillman-Grassl and RSK bijections, see e.g. [137, §9.1]. Application to sampling of this bijection have been analyzed in [19]. On the other hand, there is strong 
evidence that the RSK-based algorithms cannot be improved. While we are far from proving this, let us note that in [140] we show linear-time reductions between all major bijections in the area, so a speedup of one of them implies a speedup of all.

The remarkable Krattenthaler bijection allows enumerations of solid partitions that fit into $[n \times n \times n]$ box [105]. This bijection is based on top of the NPS algorithm, which has also been recently analyzed [129, 157. Curiously, there are no analogous results in $d \geq 4$ dimensions, making counting such $d$-dimensional partitions an open problem (cf. [77]).

3.4. Complexity of bijections. Let us now discuss the questions $\left(2^{\prime}\right)$ in the introduction, about the nature of bijections $\varphi: \mathcal{P}_{n} \rightarrow \mathcal{Q}_{n}$ from an algorithmic point of view.

Let $\left|\mathcal{P}_{n}\right|=\left|\mathcal{Q}_{n}\right|$ and think of $\varphi$ as a map. We require both $\varphi$ and $\varphi^{-1}$ to be computable in polynomial time 6 If that's all we want, it suffices to show that $\mathcal{P}_{n}$ and $\mathcal{Q}_{n}$ can be enumerated in polynomial time. Here by enumerated we mean a bijection $\phi: \mathcal{P}_{n} \rightarrow\left\{1, \ldots,\left|\mathcal{P}_{n}\right|\right\}$, where both $\phi$ and $\phi^{-1}$ are computable in polynomial time.

For example, the dynamic programming plus divide-and-conquer proves that the sets of partitions $\mathcal{P}_{n}$ and $\mathcal{Q}_{n}$ on both sides of the Rogers-Ramanujan identity (3.1), can be enumerated in poly $(n)$ time. This gives a bijection $\varphi_{n}: \mathcal{P}_{n} \rightarrow \mathcal{Q}_{n}$, proving the identity. But since proving validity of such construction would require prior knowledge of $\left|\mathcal{P}_{n}\right|=\left|\mathcal{Q}_{n}\right|$, from a combinatorial point of view this bijection is unsatisfactory.

Alternatively, one can think of a bijection as an algorithm that computes a given map $\varphi_{n}$ as above in poly $(n)$ time. This is a particularly unfriendly setting, as one would essentially need to prove new lower bounds in complexity. Worse, we proved in [103] that in some cases O'Hara's algorithm requires superpolynomial time, while the map given by the algorithm can be computed in poly $(n)$ time using integer programming. Since this is the only nice bijective proof of the Andrews identities that we know (see [137]), this suggests that either we don't understand the nature of these identities or have a very restrictive view of what constitutes a combinatorial bijection. Or, perhaps, the complexity approach is simply inapplicable in this combinatorial setting.

There are other cases of unquestionably successful bijections which are inferior to other algorithms from the complexity point of view. For example, stretching the definitions a bit, Wilson's LERW algorithm for generating random (directed) spanning trees uses exponential time on directed graphs [175], while a straightforward algorithm based on the matrix-tree theorem is polynomial, of course.

Finally, even when the bijection is nice and efficient, it might still have no interesting properties, so the only application is the proof of the theorem. One example is an iterative bijection for the Rogers-Ramanujan identity (3.1) which is implied by the proof in [24]. It is unclear if it respects any natural statistics which would imply a stronger result. Thus, it is presented in 24] in the form of a combinatorial proof to make the underlying algebra clear.

3.5. Probabilistic/asymptotic approach. Suppose both sets of combinatorial objects $\mathcal{P}_{n}$ and $\mathcal{Q}_{n}$ have well-defined limit shapes $\pi$ and $\omega$, as $n \rightarrow \infty$. Such limit shapes exist for various families of trees [50], graphs [116], partitions [44], permutations [87], solid partitions [132], Young tableaux [153], etc] For a sequence $\left\{\varphi_{n}\right\}$ of bijections $\varphi_{n}: \mathcal{P}_{n} \rightarrow \mathcal{Q}_{n}$, one can ask about the limit bijection $\Phi: \pi \rightarrow \omega$, defined as $\lim _{n \rightarrow \infty} \varphi_{n}$. We can then require that $\Phi$

\footnotetext{
${ }^{6}$ Here we are trying to avoid having one-way functions, which play an important role in cryptography, but are distracting in this setting.

${ }^{7}$ Here the notion of a "limit shape" is used very loosely, as it means very different things in each case.
} 
satisfies certain additional structural properties. This is the approach taken in [136] to prove the following result:

Theorem 3.1. The Rogers-Ramanujan identity (3.1) has no geometric bijection.

Here the geometric bijections are defined as compositions of certain piecewise $\operatorname{GL}(2, \mathbb{Z})$ maps acting on Ferrers diagrams, which are viewed as subsets of $\mathbb{Z}^{2}$. We first prove that the limits of such bijections are asymptotically stable, i.e. act piecewise linearly on the limit shapes. The rest of the proof follows from existing results on the limit shapes $\pi$ and $\omega$ on both sides of (3.1), which forbid a piecewise linear map $\Phi: \pi \rightarrow \omega$, see [44].

The next story is incomplete, yet the outlines are becoming clear. Let $\operatorname{ASM}(n)$ be the number of alternating sign matrices of order $n$, defined as the number of $n \times n$ matrices where every row has entries in $\{0, \pm 1\}$, with row and column sums equal to 1 , and all signs alternate in each row and column. Let $\operatorname{FSLT}(n)$ be the number of the fully symmetric lozenge tilings, defined as lozenge tilings of the regular $2 n$-hexagon with the full group of symmetries $D_{6}$. Such tilings are in easy bijection with solid partitions that fit into a $[2 n \times 2 n \times 2 n]$ box, have full group of symmetries $S_{3}$, and are self-complementary within the box (cf. $\sqrt{3.3}$ ). Finally, let $\operatorname{TSPP}(n)$ be the number of triangular shifted plane partitions defined as plane partitions $\left(b_{i j}\right)_{1 \leq i \leq j}$ of shifted shape $(n-1, n-2, \ldots, 1)$, and entries $n-i \leq b_{i j} \leq n$ for $1 \leq i \leq j \leq n-1$.

The following identity is justly celebrated:

$$
\operatorname{ASM}(n)=\operatorname{FSLT}(n)=\operatorname{TSPP}(n)=\frac{1 ! 4 ! 7 ! \cdots(3 n-2) !}{n !(n+1) ! \cdots(2 n-1) !}
$$

See [29] for the history of the problem and [158, A005130] for further references.

Now, the second equality is known to have a bijective proof [124]. The third equality is a major open problem (see below).

Claim 3.2. The equality $\operatorname{ASM}(n)=\operatorname{FSLT}(n)$ has no geometric bijection.

We now know (conjecturally) what the frozen regions in each case are: the circle for FSLTs and a rather involved sextic equation for ASMs. The latter is an ingenuous conjecture in [36] (see also [37), while the former is a natural conjecture about the Arctic Circle which remains when the symmetries are introduced (cf. [143]) 8 We are not sure in this case what do we mean by a "geometric bijection". But any natural definition should imply that the two shapes are incompatible. It would be interesting to formalize this even before both frozen regions are fully established.

There is another aspect of this asymptotic approach, which allows us to distinguish between different equinumerous collections of combinatorial objects with respect to some (transitive) notions of a "good" (canonical) bijection, and thus divide them into equivalence classes. This method would allow us to understand the nature of these families and ignore superficial differences within the same class.

The prototypical example of this is a collection of over 200 objects enumerating Catalan numbers [162, but there are other large such collections: for Motzkin numbers, Schröder numbers, Euler numbers (1.11), etc. A natural approach would be to use the symmetry properties or the topology, but such examples are rare (see, however, [1] and [172] for two "canonical" bijections between Catalan objects).

In [125], we studied the limit averages of permutation matrices corresponding to $\mathrm{A}_{n}(\mathcal{F})$. We showed that the limit surfaces corresponding to $\mathrm{A}_{n}(123)$ and $\mathrm{A}_{n}(213)$ are quite different,

\footnotetext{
${ }^{8}$ While the frozen region hasn't been established for FSLTs, it is known that if exists it must be a circle (Greta Panova, personal communication).
} 
even though their sizes are Catalan numbers (see also [86, 119]). This partly explains a well known phenomenon: there are at least nine(!) different explicit bijections between these two families, see [99], each with its own special properties. Evidently, there is simply no "canonical" bijection in this case. See also [7, 46] for the asymptotic analysis of two other interesting Catalan families.

3.6. Open problems on bijections. In theory, having a direct bijection should be an exception, not a rule, since in most cases the algebraic tools are simply more powerful. In practice, combinatorialists tend to be fascinated with basic structures reflecting certain most fundamental symmetries, where the bijections are abound. There are, however, a few notable examples where the bijections have been sought for years, sometimes for decades, with little hope of success. Below is a very short list from the many remarkable identities.

(1) Dyson's rank problem. Prove bijectively: $p_{0}(5 k-1)=p_{1}(5 k-1)=p_{2}(5 k-1)$. Here $p_{i}(n)$ is the number of partitions $\lambda \vdash n$ such that $\lambda_{1}-\lambda_{1}^{\prime}=i \bmod 5$, see [52]. Note that asymptotic methods are inapplicable here (for the purposes of proving non-existence of such bijection), but there is an elegant algebraic proof [68].

(2) Triangular shifted plane partition. Prove bijectively the product formula (3.2) for TSPP $(n)$. We refer to [29, 106] for more on the history and the context.

(3) Symmetry of q, t-Catalan numbers. Prove bijectively:

$$
F_{n}(x, y)=F_{n}(y, x), \quad \text { where } F_{n}(x, y)=\sum_{\pi \in \operatorname{Dyck}(n)} x^{\operatorname{area}(\pi)} y^{\operatorname{dinv}(\pi)}
$$

where $\operatorname{Dyck}(n)$ is the set of Dyck paths of length $2 n$ and two statistics are defined in [81.

(4) Linear extensions of Selberg posets 9 Let $\mathrm{P}(a, b, c)$ be a poset defined as in Figure 2, Let $e(\mathrm{Q})$ denote the number of linear extensions of the poset Q. Prove bijectively:

$$
e(\mathrm{P}(a, b, c))=\frac{(a+c) !(b+c) !(2 a+2 b+2 c+1) !}{a ! b ! c !(a+b+c) !(a+b+2 c+1) !} .
$$

See Ex. 1.11 and 3.55 in [160] for generalization and connection to the Selberg integral.

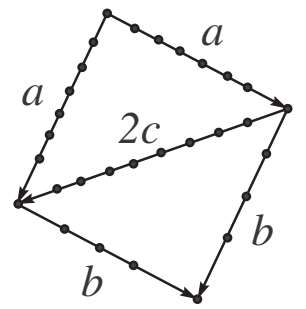

Figure 2. Selberg poset $P(6,3,4)$.

(5) Standard Young tableaux of skew shape. Prove bijectively:

$$
\# \operatorname{SYT}\left((3 a)^{2 a}(2 a)^{a} / a^{a}\right)=\frac{\left(7 a^{2}\right) ! \Phi(a)^{5} \Phi(5 a)}{\Phi(2 a)^{2} \Phi(6 a)},
$$

where $\Phi(n)=1 ! \cdot 2 ! \cdots(n-1)$ ! is the superfactorial [158, A000178. This is a special case of two large series of shapes recently discovered in [98] and [128. One can also view $\operatorname{SYT}(\lambda)$ as

\footnotetext{
${ }^{9}$ We introduced Selberg posets in a 2003 NSF proposal, see solution to [160, Exc. 3.55].
} 
linear extensions of the corresponding poset, and in this case it also can be derived from the Selberg integral [98].

Remark 3.3. Let us note that posets in (4) and (5) have dimension two, meaning they are defined by a set of points $\left\{\left(x_{1}, y_{1}\right), \ldots,\left(x_{n}, y_{n}\right)\right\} \subset \mathbb{R}^{2}$, with $\left(x_{i}, y_{i}\right) \preccurlyeq\left(x_{j}, y_{j}\right)$ when $x_{i} \leq x_{j}$ and $y_{i} \leq y_{j}$. The formulas above allow computation of the number of linear extensions in polynomial time. Perhaps surprisingly, many other sequences in this paper also count linear extensions of 2-dimensional posets: binomial coefficients $\left(\begin{array}{l}n \\ k\end{array}\right)$, Catalan numbers $C_{n}$, Fibonacci numbers $F_{n}$, Euler numbers $E_{n}$, etc. (see e.g. [127, 128]).

In fact, Feit's determinant formula [56] for $\operatorname{SYT}(\lambda / \mu)$ implies that the number of linear extensions can be computed in $\operatorname{poly}(n)$ time for all skew shapes, where $n=|\lambda / \mu|$. This raises a question if $e(\mathrm{P})$ can be computed in polynomial time for other "natural" families of shapes, e.g. cross shapes $\operatorname{Cr}(a, b)$ defined as $[b \times b]$ minus four corner $[a \times a]$ squares, $b>2 a$ (cf. [2]). Finally, let us mention that computing $e(\mathrm{P})$ is \#P-complete for general 2-dimensional posets [45].

\section{Combinatorial interpretations}

4.1. Complexity setting. Let $\mathcal{A}=\cup_{n} \mathcal{A}_{n}$ be a family of combinatorial objects, which means that membership in $\mathcal{A}_{n}$ can be decided in poly $(n)$ time (see 81.3 ). Let $f: \mathcal{A} \rightarrow \mathbb{N}$ be a computable function, which we assume to be at most exponential: $f(X) \leq e^{C n^{a}}$ for all $X \in \mathcal{A}_{n}$, and some $C, a>0$. Let

$$
\mathcal{P}=\bigcup_{X \in \mathcal{A}} \mathcal{P}_{X}
$$

be a family of combinatorial objects parameterized by $\mathcal{A}$, such that $\left|\mathcal{P}_{X}\right|=f(X)$. We then say that $\mathcal{P}$ is a combinatorial interpretation of $f$.

In the language of computational complexity, if $f$ has a combinatorial interpretation, then the problem of computing $f(X)$ is in \#P. Similarly, suppose $f_{1}$ and $f_{2}$ have combinatorial interpretations, and that $f=f_{1}-f_{2}$. Then the problem of computing $f(X)$ is in the complexity class GapP, defined exactly to be the class of differences of two \#P functions. It is typical in combinatorics to consider a nonnegative GapP function and ask if it is in \#P (see examples below).

4.2. Kronecker coefficients. One especially notable case of a combinatorial interpretation is the problem of computing the Kronecker coefficients of the symmetric group, which are defined by

$$
\chi^{\lambda} \cdot \chi^{\mu}=\sum_{\nu \vdash n} g(\lambda, \mu, \nu) \chi^{\nu}, \quad \text { where } \lambda, \mu \vdash n,
$$

and $\chi^{\lambda}, \chi^{\mu}, \chi^{\nu}$ are irreducible characters of $S_{n}$.

Open Problem 4.1. Find a combinatorial interpretation for the Kronecker coefficients

$$
\{g(\lambda, \mu, \nu), \lambda, \mu, \nu \vdash n\} .
$$

This problem was introduced by F. D. Murnaghan in 1938 and it has been studied extensively in recent years, both as a problem in algebraic combinatorics and in connection to the geometric complexity theory. We refer to [18, 139] for details and further references. 
Now, it was shown by Bürgisser and Ikenmeyer [31] that computing $g(\lambda, \mu, \nu)$ is in GapP. An elementary proof of this is given in [139]:

$$
g(\lambda, \mu, \nu)=\sum_{\sigma, \omega, \pi \in S_{\ell}} \operatorname{sgn}(\sigma \omega \pi) \cdot \mathrm{CT}(\lambda-\sigma+\mathbf{1}, \mu-\omega+\mathbf{1}, \nu-\pi+\mathbf{1}),
$$

where $\mathbf{1}=(1, \ldots, 1)$, the number of rows $\ell(\lambda), \ell(\mu), \ell(\nu) \leq \ell$, and $\mathrm{CT}(\alpha, \beta, \gamma)$ is the number of 3 -dimensional contingency tables with 2-dimensional sums $\alpha, \beta, \gamma$. The problem is known to be \#P-hard [31]. In a recent remarkable development, it was shown in [89] that the positivity decision problem $g(\lambda, \mu, \nu)>$ ? 0 is NP-hard, so a combinatorial interpretation would imply that this problem is NP-complete.

4.3. Hamiltonian cycles in cubic graphs. The Kronecker coefficients problem discussed above is fundamentally an issue of constructive subtraction encapsulated by the GapP complexity class. There is a similar constructive division issue, which is rare in complexity theory, but routine in combinatorics, where many proofs are based on double counting. For example, both Joyal's and Pitman's proofs of the Cayley formula (1.12) are by double counting (see the first and the fourth proof in [4, Ch. 32]).

Theorem 4.2 (Smith and Tutte [167]). Let e be an edge in a cubic graph G. Then the number $N_{e}(G)$ of Hamiltonian cycles in $G$ containing $e$, is always even.

The original proof is an elegant double counting argument modulo 2. This suggests the following open problem, 10

Open Problem 4.3. Find a combinatorial interpretation for $N_{e}(G) / 2$.

For example, when $G$ is uniquely 3-edge-colorable, we have $N_{e}(G)=2$ and the problem is easy. The following constructive proof of Theorem 4.2 is due to Price [147] and Thomason [166]:

Proof of Theorem 4.2. Consider the set of Hamiltonian paths $P$ in $G$ with $e$ as the initial step. Initially, let $P$ be a Hamiltonian cycle in $G$ minus edge $e_{1}$ adjacent to $e$. The endpoint of $P$ that is not in $e$ has two adjacent edges $e_{1}, e_{2} \notin P$. Extend $P$ by $e_{2}$ and remove a uniquely determined edge which creates a new path $P^{\prime}$. This transformation is called Pósa rotation. Repeat Pośa rotations until one would be forced to remove $e$, at which point you get a new Hamiltonian cycle. This defines a pairing of all Hamiltonian cycles.

If the Price-Thomason algorithm defined in the proof above were polynomial, we would have a solution for the open problem. Indeed, for a Hamiltonian cycle in $G$ we accept it if and only if it is lexicographically smaller than the Hamiltonian cycle it is paired with by the algorithm. Unfortunately, the algorithm is exponential [107] (see also [33]).

Note the strong similarities with the involution principle (see 3.2 ) and the division by two [47] which are all based on the same principle. The problem and the proof above are variations on Another Hamiltonian CyCle problem, which is in the complexity class PPA conjectured to be outside of $P$, see [144, Ex. 10.7] and [126, §6.7.2]. This suggests that the open problem cannot be resolved in full generality (in a mathematical sense), but perhaps some

We should mention that in some cases the constructive division problem has been resolved. Most notably, the Ramanujan congruences modulo 5 and 7, see [84, §19.12], were combinatorially interpreted by Dyson, with a proof of his conjectural combinatorial interpretation

\footnotetext{
${ }^{10}$ This problem was suggested to us by Peter Shor https://tinyurl.com/y7yafneh
} 
given later by Atkin and Swinnerton-Dyer. See Dyson's rank problem in 33.6 and an alternative statistics called crank, introduced in [10] for congruences modulo 11, leading to many generalizations [120].

4.4. Combinatorial interpretation of sequences. Let $\left\{a_{n}\right\}$ be a nonnegative integer sequence that has a Wilfian formula of type (W1), i.e. can be computed in poly $(n)$ time. Then $a_{n}$ has a trivial combinatorial interpretation: integers $\left\{1, \ldots, a_{n}\right\}$. This means that for sequences we need a different notion.

Let $\mathcal{P}=\cup \mathcal{P}_{n}$ be the set of combinatorial objects, s.t. $\left|\mathcal{P}_{n}\right|=a_{n}$ for all $n$. We say that $\mathcal{P}$ gives a combinatorial interpretation for $\left\{a_{n}\right\}$ of type:

(C1) if membership in $\mathcal{P}_{n}$ can be decided in $O(\log n)$ space.

For example, the $0-1$ ballot sequences of length $2 n$ with $n$ ones and $n$ zeroes give a combinatorial interpretation of the Catalan number $C_{n}$. Indeed, to verify membership it suffices to have two counters: \#1's and \#0's, which require $O(\log n)$ space.

Note that a combinatorial interpretation of type $(\mathrm{C} 1)$ is a property not only of the objects in $\mathcal{P}$, but also of their presentation. For example, permutations $\sigma \in S_{n}$ in their natural representation are not of type $(\mathrm{C} 1)$. To make them of type $(\mathrm{C} 1)$ one can represent them with a permutation matrix.

4.5. Super Catalan numbers. The strange case of super Catalan numbers shows both advantage and disadvantage of the complexity approach. They are defined as follows:

$$
S(m, n):=\frac{(2 m) !(2 n) !}{m ! n !(m+n) !} .
$$

These were defined by E. Catalan in 1874, who discovered that they are integers. This follows easily from the divisibility properties of the factorials as well as the von Szily identity (1894):

$$
S(m, n)=\sum_{k}(-1)^{k}\left(\begin{array}{c}
2 m \\
m+k
\end{array}\right)\left(\begin{array}{c}
2 n \\
n+k
\end{array}\right) .
$$

Note that $S(1, n) / 2=C_{n}$ is the usual Catalan number.

The problem of finding a combinatorial interpretation was posed by Gessel in [72. Such an interpretation is known for $m \leq 3$ and for $|m-n| \leq 3$, see [35, 74] (see also [8, 155]). Yet, from a computational complexity point of view, Gessel also suggested the proof idea.

Theorem 4.4. The numbers $S(m, n)$ have a combinatorial interpretation of type $(\mathrm{C} 1)$.

Proof. Following Gessel [72], we have

$$
S(m, m+\ell)=\sum_{k} 2^{\ell-2 k}\left(\begin{array}{c}
\ell \\
2 k
\end{array}\right) S(m, k) .
$$

Together with the symmetry $S(m, n)=S(n, m)$ and the initial condition $S(0,0)=1$, this recursively defines a combinatorial interpretation. Indeed, at each recursive step a $0-1$ word of length $(\ell-2 k)+\ell \leq 2 \ell$ is added. Since $k \leq \ell / 2$, the number of steps to compute $S(m, n)$, where $m \leq n$, is at most $O(n \log n)$. All such words put together, this gives a word of length $O\left(n^{2} \log n\right)$. The description of each step can be verified in $O(\log \ell)$ space, giving the total space $O(\log m+\log n)$, as desired. 
Remark 4.5. Finding a combinatorial interpretation for the super Catalan numbers $S(m, n)$ is repeatedly stated as an open problem, see e.g. [159, Exc. 66]. Gessel writes: "it remains to be seen whether (4.1) can be interpreted in a 'natural' way" [72. This was later echoed in [74: "Formula (4.1) allows us to construct recursively a set of cardinality $S(m, n)$, but it is difficult to give a natural description of this set."

Given the argument above, we are somewhat puzzled as to what exactly is an open problem, and remain deeply dissatisfied with the "you know it when you see it" answer. As best as we can tell, the unwritten goal is to represent $S(m, n)$ as the number of certain (collections of) lattice paths, which would allow an easy inductive proof and passing to an existing $q$-analogue [171].

Alternatively, one can ask about a combinatorial interpretation coupled with a double counting argument elucidating the product formula for $S(m, n)$, as in [155]. Such notion would be more restrictive, of course, and thus harder to obtain. It would also be a more exciting discovery.

4.6. Gessel sequence. In [73], the author defined the following Gessel sequence:

$$
b_{n}:=2 \cdot 5^{n}-(3+4 i)^{n}-(3-4 i)^{n}, \quad \text { where } i=\sqrt{-1},
$$

see also [158, A250102]. Note that $b_{n} \in \mathbb{Z}$ since

$$
b_{n}=2 \cdot 5^{n}-2 \sum_{r}(-1)^{r}\left(\begin{array}{c}
n \\
2 r
\end{array}\right) 3^{n-2 r} 4^{2 r}
$$

and that $b_{i} \geq 0$ since $|3 \pm 4 i|=5$. Note also that $\left\{b_{n}\right\}$ is C-recursive since

$$
B(t):=\sum_{n=0}^{\infty} b_{n} t^{n}=\frac{16 t(1+5 t)}{(1-5 t)\left(1+6 t+25 t^{2}\right)}
$$

This is an example of a C-recursive nonnegative sequence without an easy combinatorial interpretation.

Conjecture 4.6 (cf. [73]). Sequence $\left\{b_{n}\right\}$ has a combinatorial interpretation of type (C1).

Let us briefly explain the significance of the sequence $\left\{b_{n}\right\}$. The class $\mathcal{R}$ of $\mathbb{N}$-rational functions is defined to be the smallest class of GFs $F(t)=a_{0}+a_{1} t+a_{2} t^{2}+\ldots$, such that

(1) $0, t \in \mathcal{R}$,

(2) $F_{1}, F_{2} \in \mathcal{R} \quad \Longrightarrow \quad F_{1}+F_{2}, F_{1} \cdot F_{2} \in \mathcal{R}$,

(3) $F \in \mathcal{R}, F(0)=0 \quad \Longrightarrow 1 /(1-F) \in \mathcal{R}$.

Clearly, $\mathcal{R} \subseteq \mathbb{Z}(t) \cap \mathbb{N}[[t]]$. This is a class of GFs for the number of words in regular languages. Equivalently, this is a class of GFs for the number of accepted paths by a finite-state automaton (FSA). The Berstel-Soittola theorem gives necessary and sufficient conditions for a nonnegative GF to be in $\mathcal{R}$ [16] (see also [73]). The corresponding sequences are exactly those that have a combinatorial interpretation $\mathcal{P}$, s.t.

(C2) membership in $\mathcal{P}_{n}$ can be decided in $O(1)$ space.

In the case of the Gessel sequence, the Berstel-Soittola theorem implies that $B(t) \notin \mathcal{R}$. This implies that $\left\{b_{n}\right\}$ has no combinatorial interpretation of type (C2), i.e. cannot be described by a FSA, thus making Conjecture 4.6 more challenging.

Remark 4.7. The (metamathematical) Schützenberger principle states that all combinatorial sequences with rational GFs must be $\mathbb{N}$-rational, see [17, p. 149]. This all depends on the meaning of the word "combinatorial", of course. Philosophy aside, we believe the conjecture above will be resolved positively, and plan to return to this problem in the near future. 
Remark 4.8. For general C-recursive integer sequences $\left\{a_{n}\right\}$, finding a combinatorial interpretation is related to the classical Skolem problem of deciding if $a_{n}=0$ for some $n$. Skolem's problem is known to be NP-hard, but not known to be decidable except for some special cases, see 5, 133. Since $\left\{a_{n}^{2}\right\}$ is also C-recursive, having a combinatorial interpretation for $\left\{a_{n}^{2}\right\}$ would not be a contradiction to undecidability, but would make it similarly curious as ReCtangular Tileability, see $\lcm{1.4}$.

4.7. Unimodality of $q$-binomial coefficients. A sequence $\left(a_{0}, a_{1}, \ldots, a_{n}\right)$ is called unimodal if for some $m$ we have

$$
a_{0} \leq a_{1} \leq \cdots \leq a_{m} \geq a_{m+1} \geq \cdots \geq a_{n} .
$$

Whenever a combinatorial sequence is proved unimodal, one can ask for a combinatorial interpretation of $\left\{a_{k}-a_{k-1}, 1 \leq k \leq m\right\}$ and $\left\{a_{k}-a_{k+1}, m \leq k \leq n-1\right\}$. For example, for $a_{k}=\left(\begin{array}{l}n \\ k\end{array}\right)$, unimodality can be checked directly. The differences $\left(\begin{array}{l}n \\ k\end{array}\right)-\left(\begin{array}{c}n \\ k-1\end{array}\right), 1 \leq k \leq n / 2$ are the ballot numbers, generalizing Catalan numbers.

The q-binomial (Gaussian) coefficients are defined as:

$$
\left(\begin{array}{c}
m+\ell \\
m
\end{array}\right)_{q}=\frac{\left(q^{m+1}-1\right) \cdots\left(q^{m+\ell}-1\right)}{(q-1) \cdots\left(q^{\ell}-1\right)}=\sum_{k=0}^{\ell m} p_{k}(\ell, m) q^{k} .
$$

Sylvester's theorem establishes unimodality of the sequence

$$
p_{0}(\ell, m), p_{1}(\ell, m), \ldots, p_{\ell m}(\ell, m) .
$$

This celebrated result was first conjectured by Cayley in 1856, and proved by Sylvester using invariant theory in a pioneering 1878 paper [164] (see [131, 138, 146, 148, for modern proofs).

Theorem 4.9 (Pak-Panova, 2015). Fix $\ell, m \geq 1$. The sequence

$$
\left\{p_{k}(\ell, m)-p_{k-1}(\ell, m), 1 \leq k \leq \ell m / 2\right\}
$$

has a combinatorial interpretation of type (C1) 11

Note that the sequence in the theorem is a special case of Kronecker coefficients:

$$
p_{k}(\ell, m)-p_{k-1}(\ell, m)=g\left(m^{\ell}, m^{\ell},(m \ell-k, k)\right) .
$$

In a roundabout way, the technical difficulties involved in the proof of Theorem 4.9 suggest that Open Problem 4.1 is unlikely to have a easy solution. Moreover, since the combinatorial interpretation of Kronecker coefficients in the theorem is in terms of certain partition-labeled trees, it is unlikely that $g(\lambda, \mu, \nu)$ in general can be expressed as the number of certain Young tableaux (cf. [18]).

Unimodality and related log-concavity problems are plentiful in combinatorics, with many connections and applications to other fields; see e.g. 28, for an introduction. While occasionally the proofs are elegant combinatorial constructions (see e.g. [82, 104]), most of them are rather difficult and technical, involving fundamentally non-combinatorial tools. Thus, for example, it would be unreasonable to expect a direct combinatorial proof of log-convexity of the partition function:

$$
p(n-1) p(n+1) \geq p(n)^{2} \text { for all } n \geq 26,
$$

\footnotetext{
${ }^{11}$ This combinatorial interpretation is based on O'Hara's identity 131 and is quite involved. We give it on p. 9 in https://tinyurl.com/ydemhyf5.
} 
see [43]. Similarly, it would be unreasonable to expect a combinatorial proof of the AdiprasitoHuh-Katz theorem on log-concavity of $\left\{a_{k}(G)\right\}$, where $G=(V, E)$ is a simple graph and $a_{k}(G)$ is the number of spanning forests in $G$ with $k$ edges [3].

\section{Final Remarks}

5.1. Enumerative combinatorics is so enormous in both range and scope, that we touched upon very few themes. If one is to summarize our choices, we tried to explain how to ask a good question on the subject. This includes both the types of questions one can ask from the complexity point of view, as well as the sources of combinatorial sequences and combinatorial objects to study.

5.2. This paper can be viewed as a technical followup to an elegant, refreshingly opinionated and very accessible introductory article [179]. To understand the state of art of Enumerative Combinatorics, we refer to an excellent monograph [160], which is remarkable in both the content (check all the exercises!) and presentation style.

For Computational Complexity definitions and the background, we recommend [126] as a fun and accessible introductory textbook. Other good options include: [75, 144] are thorough monographs, [1] is a beautifully written up to date introductory survey, and [173] is a remarkable recent monograph-length survey of the area with a lot of advanced mathematical content.

As of now, the complexity approach pioneered by Wilf in [174 has yet to fully blossom into a research area of Computational Combinatorics 12 However, the fundamentals of computational complexity are clearly as important as basic algebra and probability. As we tried to explain on these pages, this computational approach can change your vision of the area and guide your understanding.

Acknowledgements. We are very grateful to Matthias Aschenbrenner, Sasha Barvinok, Artëm Chernikov, Persi Diaconis, Sam Dittmer, Jacob Fox, Éric Fusy, Bon-Soon Lin, Pasha Pylyavskyy, Vic Reiner, Richard Stanley and Jessica Striker for helpful remarks and interesting conversations.

We are thankful to Michael Albert, Allen Gehret, Fredrik Johansson, Alex Mennen, Marni Mishna, Cris Moore, Jay Pantone, Martin Rubey, Gilles Schaeffer, Jeff Shallit and Vince Vatter for reading the paper, their comments and useful remarks. Ira Gessel kindly suggested the proof of Theorem 1.4

Special thanks to Stephen DeSalvo, Scott Garrabrant, Alejandro Morales, Danny Nguyen, Greta Panova, Jed Yang and Damir Yeliussizov for many collaborations and numerous discussions, some of which undoubtedly influenced the presentation above. The author was partially supported by the NSF.

\footnotetext{
${ }^{12}$ See our blog post https://igorpak.wordpress.com/2012/07/25/computational-combinatorics/
} 


\section{REFERENCES}

[1] S. Aaronson, $\mathrm{P} \stackrel{?}{=} \mathrm{NP}$, in Open problems in mathematics, Springer, New York, 2016, 1-122.

[2] R. Adin and Y. Roichman, Standard Young tableaux, in Handbook of enumerative combinatorics, CRC Press, Boca Raton, FL, 2015, 895-974.

[3] K. Adiprasito, J. Huh and E. Katz, Hodge theory for combinatorial geometries; arXiv: 1511.02888.

[4] M. Aigner and G. M. Ziegler, Proofs from The Book (Fifth ed.), Springer, Berlin, 2014.

[5] S. Akshay, N. Balaji and N. Vyas, Complexity of restricted variants of Skolem and related problems, in Proc. 42nd MFCS (2017), Dagstuhl, Germany, 78:1-78:14.

[6] M. H. Albert, C. Homberger, J. Pantone, N. Shar and V. Vatter, Generating permutations with restricted containers; arXiv: 1510.00269

[7] D. Aldous, Triangulating the circle, at random, Amer. Math. Monthly 101 (1994), 223-233.

[8] E. Allen and I. Gheorghiciuc, A weighted interpretation for the super Catalan numbers, J. Integer Seq. 17 (2014), no. 10, Art. 7, 9 pp.

[9] G. E. Andrews, Partitions, in Combinatorics: ancient and modern (R. Wilson and J. J. Watkins, eds.), Oxford Univ. Press, Oxford, 2013, 205-229.

[10] G. E. Andrews and F. G. Garvan, Dyson's crank of a partition, Bull. AMS 18 (1988), 167-171.

[11] D. Armstrong, C. Stump and H. Thomas, A uniform bijection between nonnesting and noncrossing partitions, Trans. AMS 365 (2013), 4121-4151.

[12] R. Arratia and S. DeSalvo, Probabilistic divide-and-conquer, Combin. Probab. Comput. 25 (2016), 324351.

[13] L. Babai, Automorphism groups, isomorphism, reconstruction, in Handbook of combinatorics, Vol. 2, Elsevier, Amsterdam, 1995, 1447-1540.

[14] L. Babai, Graph isomorphism in quasipolynomial time, arXiv:1512.03547; extended abstract in Proc. 48th STOC (2016), ACM, New York, 2016, 684-697.

[15] O. Bernardi, M. Bousquet-Mélou and K. Raschel, Counting quadrant walks via Tutte's invariant method; arXiv: 1708.08215.

[16] J. Berstel and C. Reutenauer, Rational series and their languages, Springer, Berlin, 1988.

[17] J. Berstel and C. Reutenauer, Noncommutative rational series with applications, Cambridge Univ. Press, Cambridge, 2011.

[18] J. Blasiak, Kronecker coefficients for one hook shape, Sém. Lothar. Combin. 77 (2017), Art. B77c, 40 pp.

[19] O. Bodini, É. Fusy and C. Pivoteau, Random sampling of plane partitions, Combin. Probab. Comput. 19 (2010), 201-226.

[20] M. Bóna, Exact enumeration of 1342-avoiding permutations, J. Combin. Theory, Ser. A 80 (1997), $257-272$.

[21] M. Bodirsky, É. Fusy, M. Kang, and S. Vigerske, Boltzmann samplers, Pólya theory, and cycle pointing, SIAM J. Comput. 40 (2011), 721-769.

[22] A. Bostan, M. Bousquet-Mélou, M. Kauers and S. Melczer, On 3-dimensional lattice walks confined to the positive octant, Ann. Comb. 20 (2016), 661-704.

[23] A. Bostan, K. Raschel and B. Salvy, Non-D-finite excursions in the quarter plane, J. Combin. Theory, Ser. A 121 (2014), 45-63.

[24] C. Boulet and I. Pak, A combinatorial proof of the Rogers-Ramanujan and Schur identities, J. Combin. Theory, Ser. A 113 (2006), 1019-1030.

[25] M. Bousquet-Mélou, Rational and algebraic series in combinatorial enumeration, in Proc. ICM, Vol. III, EMS, Zürich, 2006, 789-826.

[26] M. Bousquet-Mélou and M. Mishna, Walks with small steps in the quarter plane, in Algorithmic probability and combinatorics, AMS, Providence, RI, 2010, 1-39.

[27] M. Bousquet-Mélou and G. Schaeffer, Walks on the slit plane, Probab. Theory Related Fields 124 (2002), 305-344.

[28] F. Brenti, Unimodal, log-concave, and Pólya frequency sequences in combinatorics, Mem. AMS, No. 413, 1989, 106 pp.

[29] D. M. Bressoud, Proofs and Confirmations, Cambridge Univ. Press, Cambridge, UK, 1999.

[30] D. M. Bressoud and D. Zeilberger, Bijecting Euler's partitions-recurrence, Amer. Math. Monthly 92 (1985), 54-55.

[31] P. Bürgisser and C. Ikenmeyer, The complexity of computing Kronecker coefficients, in Proc. DMTCS, Nancy, 2008, 357-368. 
[32] N. Calkin, J. Davis, K. James, E. Perez and C. Swannack, Computing the integer partition function, Math. Comp. 76 (2007), 1619-1638.

[33] K. Cameron, Thomason's algorithm for finding a second Hamiltonian circuit through a given edge in a cubic graph is exponential on Krawczyk's graphs, Discrete Math. 235 (2001), 69-77.

[34] R. Canfield, S. Corteel and P. Hitczenko, Random partitions with non-negative $r$-th differences, Adv. Appl. Math. 27 (2001), 298-317.

[35] X. Chen and J. Wang, The super Catalan numbers $S(m, m+s)$ for $s \leq 3$ and some integer factorial ratios, REU project (2012), UMN, Minneapolis, https://tinyurl.com/yculnwfz.

[36] F. Colomo and A. G. Pronko, The limit shape of large alternating sign matrices, SIAM J. Discrete Math. 24 (2010), 1558-1571.

[37] F. Colomo and A. Sportiello, Arctic curves of the six-vertex model on generic domains: the tangent method, J. Stat. Phys. 164 (2016), 1488-1523.

[38] A. Conway, A. Guttmann and P. Zinn-Justin, 1324-avoiding permutations revisited; arXiv:1709.01248.

[39] S. Corteel and C. D. Savage, Partitions and compositions defined by inequalities, Ramanujan J. 8 (2004), 357-381.

[40] R. Crandall and C. Pomerance, Prime numbers. A computational perspective (Second ed.), Springer, New York, 2005.

[41] N. G. de Bruijn and B. J. M. Morselt, A note on plane trees, J. Combin. Theory 2 (1967), 27-34.

[42] J. A. De Loera, J. Rambau and F. Santos, Triangulations, Springer, Berlin, 2008.

[43] S. DeSalvo and I. Pak, Log-concavity of the partition function, Ramanujan J. 38 (2015), 61-73.

[44] S. DeSalvo and I. Pak, Limit shapes via bijections; arXiv:1611.06073.

[45] S. Dittmer and I. Pak, Counting linear extensions of restricted posets, arXiv:1802.06312.

[46] T. Dokos and I. Pak, The expected shape of random doubly alternating Baxter permutations, Online J. Anal. Comb. 9 (2014), Paper 6, 12 pp.

[47] P. G. Doyle and J. H. Conway, Division by three; arXiv:math/0605779.

[48] T. Dreyfus, C. Hardouin and J. Roques, Hypertranscendence of solutions of Mahler equations, to appear in J. Eur. Math. Soc.; arXiv:1507.03361.

[49] T. Dreyfus, C. Hardouin, J. Roques and M. F. Singer, On the nature of the generating series of walks in the quarter plane, to appear in Invent. Math.; arXiv:1702.04696

[50] M. Drmota, Random trees, Springer, New York, 2009.

[51] P. Duchon, P. Flajolet, G. Louchard and G. Schaeffer, Boltzmann samplers for the random generation of combinatorial structures, Combin. Probab. Comput. 13 (2004), 577-625.

[52] F. J. Dyson, Some guesses in the theory of partitions, Eureka (Cambridge University) 8 (1944), 10-15; available at https://tinyurl.com/ycmaxtvf

[53] M. Elder, A. Rechnitzer, E. J. Janse van Rensburg and T. Wong, The cogrowth series for $\operatorname{BS}(N, N)$ is D-finite, Int. J. Algebra Comput. 24 (2014), 171-187.

[54] P. van Emde Boas, The convenience of tilings, in Complexity, logic, and recursion theory, Dekker, New York, 1997, 331-363.

[55] P. Erdős and A. Rényi, Asymmetric graphs, Acta Math. Acad. Sci. Hungar 14 (1963), 295-315.

[56] W. Feit, The degree formula for the skew-representations of the symmetric group, Proc. AMS 4 (1953), 740-744.

[57] S. R. Finch, Mathematical constants, Cambridge Univ. Press, Cambridge, 2003.

[58] P. Flajolet, S. Gerhold and B. Salvy, On the non-holonomic character of logarithms, powers, and the $n$-th prime function, El. J. Combin. 11 (2005), no. 2, Article 2, 16 pp.

[59] P. Flajolet and R. Sedgewick, Analytic combinatorics, Cambridge Univ. Press, Cambridge, 2009.

[60] J. Fox, Stanley-Wilf limits are typically exponential, to appear in Adv. Math.

[61] J. Freire, C. J. Klivans, P. H. Milet and N. C. Saldanha, On the connectivity of spaces of three-dimensional domino tilings; arXiv:1702.00798.

[62] H. Furstenberg, Algebraic functions over finite fields, J. Algebra 7 (1967), 271-277.

[63] É. Fusy, Counting unrooted maps using tree-decomposition, Sém. Lothar. Combin. 54A (2006), Art. B54Al, 44 pp.

[64] M. R. Garey, D. S. Johnson and R. E. Tarjan, The planar Hamiltonian circuit problem is NP-complete, SIAM J. Comput. 5 (1976), 704-714.

[65] S. Garrabrant and I. Pak, Pattern avoidance is not P-recursive; arXiv:1505.06508.

[66] S. Garrabrant and I. Pak, Words in linear groups, random walks, automata and P-recursiveness, J. Comb. Algebra 1 (2017), 127-144. 
[67] S. Garrabrant and I. Pak, in preparation.

[68] F. Garvan, D. Kim and D. Stanton, Cranks and t-cores, Invent. Math. 101 (1990), 1-17.

[69] A. M. Garsia and S. C. Milne, A Rogers-Ramanujan bijection, J. Combin. Theory, Ser. A 31 (1981), 289-339.

[70] I. M. Gessel, Problem H-187, Fibonacci Quart. 10 (1972), 417-419.

[71] I. M. Gessel, Symmetric functions and P-recursiveness, J. Combin. Theory, Ser. A 53 (1990), 257-285.

[72] I. M. Gessel, Super ballot numbers, J. Symbolic Comput. 14 (1992), 179-194.

[73] I. M. Gessel, Rational functions with nonnegative integer coefficients (talk slides), in Proc. 50th Sém. Loth. Comb. (March 2003); available at http://tinyurl.com/krlbvvl.

[74] I. M. Gessel and G. Xin, A combinatorial interpretation of the numbers $6(2 n) ! / n !(n+2)$ !, J. Integer Seq. 8 (2005), no. 2, Art. 3, 13 pp.

[75] O. Goldreich, Computational Complexity. A Conceptual Perspective, Cambridge Univ. Press, Cambridge, 2008.

[76] I. P. Goulden and D. M. Jackson, Labelled graphs with small vertex degrees and P-recursiveness, SIAM J. Alg. Disc. Methods 7 (1986), 60-66.

[77] S. Govindarajan, Notes on higher-dimensional partitions, J. Combin. Theory, Ser. A 120 (2013), 600622.

[78] R. Grigorchuk and I. Pak, Groups of intermediate growth: an introduction, Enseign. Math. 54 (2008), $251-272$.

[79] A. J. Guttmann (Ed.), Polygons, polyominoes and polycubes, Springer, Dordrecht, 2009.

[80] M. Haiman, Noncommutative rational power series and algebraic generating functions, Eur. J. Combin. 14 (1993), 335-339.

[81] J. Haglund, Catalan paths and $q$, -enumeration, in Handbook of enumerative combinatorics, CRC Press, Boca Raton, FL, 2015, 679-751.

[82] H. Han and S. Seo, Combinatorial proofs of inverse relations and log-concavity for Bessel numbers, European J. Combin. 29 (2008), 1544-1554.

[83] F. Harary and E. M. Palmer, Graphical enumeration, Academic Press, New York, 1973.

[84] G. H. Hardy and E. M. Wright, An introduction to the theory of numbers (Sixth ed.), Oxford Univ. Press, Oxford, 2008.

[85] D. Harvey, A subquadratic algorithm for computing the $n$-th Bernoulli number, Math. Comp. 83 (2014), 2471-2477.

[86] C. Hoffman, D. Rizzolo and E. Slivken, Pattern-avoiding permutations and Brownian excursion, part I: shapes and fluctuations, Random Str. Algorithms 50 (2017), 394-419.

[87] C. Hoppen, Y. Kohayakawa, C. G. Moreira, B. Ráth and R. Menezes Sampaio, Limits of permutation sequences, J. Combin. Theory, Ser. B 103 (2013), 93-113.

[88] S. P. Humphries, Cogrowth of groups and the Dedekind-Frobenius group determinant, Math. Proc. Cambridge Philos. Soc. 121 (1997), 193-217.

[89] C. Ikenmeyer, K. Mulmuley and M. Walter, On vanishing of Kronecker coefficients, Comput. Complexity 26 (2017), 949-992.

[90] C. G. J. Jacobi, Über die Differentialgleichung, welcher die Reihen $1 \pm 2 q+2 q^{4} \pm 2 q^{9}+$ etc., Crelles J. 36 (1848), 97-112.

[91] F. Johansson, Efficient implementation of the Hardy-Ramanujan-Rademacher formula, LMS J. Comput. Math. 15 (2012), 341-359.

[92] V. Kaibel and G. Ziegler, Counting lattice triangulations, in Surveys in combinatorics, Cambridge Univ. Press, Cambridge, 2003, 277-307.

[93] M. Kang and P. Sprüssel, Symmetries of Unlabelled Planar Triangulations, El. J. Combin. 25:1 (2018), paper \#P1.34, 38 pp.

[94] M. Kassabov and I. Pak, Groups of oscillating intermediate growth, Ann. Math. 177 (2013), $1113-1145$.

[95] M. Kassabov and I. Pak, Continuum many spectral radii of finitely generated groups, in preparation.

[96] R. Kenyon, An introduction to the dimer model, in ICTP Lect. Notes XVII, Trieste, 2004, 267-304.

[97] H. Kesten, Symmetric random walks on groups, Trans. AMS 92 (1959), 336-354.

[98] J. S. Kim and S. Oh, The Selberg integral and Young books, J. Combin. Theory, Ser. A 145 (2017), $1-24$.

[99] S. Kitaev, Patterns in Permutations and Words, Springer, Berlin, 2011.

[100] M. Klazar, Bell numbers, their relatives, and algebraic differential equations, J. Combin. Theory, Ser. A 102 (2003), 63-87. 
[101] M. Klazar, Some general results in combinatorial enumeration, in Proc. Permutation Patterns (St. Andrews 2007), Cambridge Univ. Press, 2010, 3-40.

[102] M. Kontsevich and D. Zagier, Periods, IHES preprint M/01/22 (May 2001), 38 pp.; available at http://tinyurl.com/k7h7gvx.

[103] M. Konvalinka and I. Pak, Geometry and complexity of O'Hara's algorithm, Adv. Applied Math. 42 (2009), 157-175.

[104] C. Krattenthaler, Combinatorial proof of the log-concavity of the sequence of matching numbers, J. Combin. Theory, Ser. A 74 (1996), 351-354.

[105] C. Krattenthaler, Another involution principle-free bijective proof of Stanley's hook-content formula, J. Combin. Theory, Ser. A 88 (1999), 66-92.

[106] C. Krattenthaler, Plane partitions in the work of Richard Stanley and his school, in The mathematical legacy of Richard P. Stanley, AMS, Providence, RI, 2016, 231-261.

[107] A. Krawczyk, The complexity of finding a second hamiltonian cycle in cubic graphs, J. Comput. Systems Sci. 58 (1999) 641-647.

[108] D. Kuksov, On rationality of the cogrowth series, Proc. AMS 126 (1998), 2845-2847.

[109] D. Kuksov, Cogrowth series of free products of finite and free groups, Glasg. Math. J. 41 (1999), 19-31.

[110] J. C. Lagarias, V. S. Miller and A. M. Odlyzko, Computing $\pi(x)$ : the Meissel-Lehmer method, Math. Comp. 44 (1985), 537-560.

[111] T. Lam, Tiling with commutative rings, Harvard College Math. Review 2 (2008), no. 1, 7 pp.

[112] D. A. Levin, Y. Peres and E. L. Wilmer, Markov chains and mixing times (Second ed.), AMS, Providence, RI, 2017.

[113] L. Levin, Universal sorting problems, Problems Inf. Transm. 9 (1973), 265-266.

[114] L. Lipshitz and L. A. Rubel, A gap theorem for power series solutions of algebraic differential equations, Amer. J. Math. 108 (1986), 1193-1214.

[115] R. J. Lipton and Y. Zalcstein, Word problems solvable in logspace, J. ACM 24 (1977), 522-526.

[116] L. Lovász, Large networks and graph limits, AMS, Providence, RI, 2012.

[117] L. Lovász and M. D. Plummer, Matching theory, North-Holland, Amsterdam, 1986.

[118] E. M. Luks, Isomorphism of graphs of bounded valence can be tested in polynomial time, J. Comput. System Sci. 25 (1982), 42-65.

[119] N. Madras and L. Pehlivan, Structure of random 312-avoiding permutations, Random Str. Algorithms 49 (2016), 599-631.

[120] K. Mahlburg, Partition congruences and the Andrews-Garvan-Dyson crank, Proc. Natl. Acad. Sci. USA 102 (2005), 15373-15376.

[121] A. Marcus and G. Tardos, Excluded permutation matrices and the Stanley-Wilf conjecture, J. Combin. Theory, Ser. A 107 (2004), 153-160.

[122] A. M. Mian and S. Chowla, The differential equations satisfied by certain functions, J. Indian Math. Soc. 8 (1944), 27-28; available at https://tinyurl.com/y7jqsk6d

[123] C. F. Miller, Decision problems for groups - survey and reflections, in Algorithms and classification in combinatorial group theory, Springer, New York, 1992, 1-59.

[124] W. H. Mills, D. P. Robbins and H. Rumsey, Self-complementary totally symmetric plane partitions, J. Combin. Theory, Ser. A 42 (1986), 277-292.

[125] S. Miner and I. Pak, The shape of random pattern-avoiding permutations, Adv. Appl. Math. 55 (2014), 86-130.

[126] C. Moore and S. Mertens, The nature of computation, Oxford Univ. Press, Oxford, 2011.

[127] A. H. Morales, I. Pak and G. Panova, Why is $\pi<2 \phi$ ?, preprint (2016); to appear in Amer. Math. Monthly; available at https://tinyurl.com/y9hlqa3y

[128] A. H. Morales, I. Pak and G. Panova, Hook formulas for skew shapes III. Multivariate and product formulas; arXiv:1707.00931.

[129] C. Neumann and R. Sulzgruber, A complexity theorem for the Novelli-Pak-Stoyanovskii algorithm, J. Combin. Theory, Ser. A 135 (2015), 85-104.

[130] J. Noonan and D. Zeilberger, The enumeration of permutations with a prescribed number of forbidden patterns, Adv. Appl. Math. 17 (1996), 381-407.

[131] K. M. O'Hara, Unimodality of Gaussian coefficients: a constructive proof, J. Combin. Theory, Ser. A 53 (1990), 29-52.

[132] A. Okounkov, Limit shapes, real and imagined, Bull. AMS 53 (2016), 187-216. 
[133] J. Ouaknine and J. Worrell, Positivity problems for low-order linear recurrence sequences, in Proc. 25th SODA (2014), ACM, New York, 366-379.

[134] I. Pak, Tile invariants: New horizons, Theor. Comp. Sci. 303 (2003), 303-331.

[135] I. Pak, Partition identities and geometric bijections, Proc. AMS 132 (2004), 3457-3462.

[136] I. Pak, The nature of partition bijections II. Asymptotic stability, preprint (2004), 32 pp; available at https://tinyurl.com/y7mduboc.

[137] I. Pak, Partition bijections, a survey, Ramanujan J. 12 (2006), 5-75.

[138] I. Pak and G. Panova, Unimodality via Kronecker products, J. Algebraic Combin. 40 (2014), 1103-1120.

[139] I. Pak and G. Panova, On the complexity of computing Kronecker coefficients, Comput. Complexity 26 (2017), 1-36.

[140] I. Pak and E. Vallejo, Reductions of Young tableau bijections, SIAM J. Discrete Math. 24 (2010), 113-145.

[141] I. Pak and J. Yang, The complexity of generalized domino tilings, El. J. Combin. 20:4 (2013), Paper 12, $23 \mathrm{pp}$

[142] I. Pak and D. Yeliussizov, in preparation.

[143] G. Panova, Lozenge tilings with free boundaries, Lett. Math. Phys. 105 (2015), 1551-1586.

[144] C. H. Papadimitriou, Computational Complexity, Addison-Wesley, Reading, MA, 1994.

[145] R. Pemantle and M. C. Wilson, Analytic combinatorics in several variables, Cambridge Univ. Press, Cambridge, 2013.

[146] M. Pouzet and I. G. Rosenberg, Sperner properties for groups and relations, European J. Combin. 7 (1986), 349-370.

[147] W. L. Price, A topological transformation algorithm which relates the Hamiltonian circuits of a cubic planar map, J. LMS 15 (1977), 193-196.

[148] R. A. Proctor, Solution of two difficult combinatorial problems with linear algebra, Amer. Math. Monthly 89 (1982), 721-734.

[149] M. van der Put and M. F. Singer, Galois theory of difference equations, Springer, Berlin, 1997.

[150] D. Revelle, Heat kernel asymptotics on the lamplighter group, El. Comm. Probab. 8 (2003), $142-154$.

[151] J. F. Ritt, Differential Algebra, AMS, New York, 1950.

[152] N. Robbins, Fibonacci partitions, Fibonacci Quart. 34 (1996), 306-313.

[153] D. Romik, The surprising mathematics of longest increasing subsequences, Cambridge Univ. Press, New York, 2015.

[154] G.-C. Rota and D. Sharp, Mathematics, Philosophy and Artificial Intelligence, dialogue in Los Alamos Science, No. 12 (Spring/Summer 1985), 94-104.

[155] G. Schaeffer, A combinatorial interpretation of super-Catalan numbers of order two, preprint (2003); https://tinyurl.com/yb7a593a

[156] G. Schaeffer, Planar maps, in Handbook of enumerative combinatorics, CRC Press, Boca Raton, FL, 2015, 335-395.

[157] C. Schneider and R. Sulzgruber, Asymptotic and exact results on the complexity of the Novelli-PakStoyanovskii algorithm, El. J. Combin. 24 (2017), Paper 2.28, 33 pp.

[158] N. J. A. Sloane, The Online Encyclopedia of Integer Sequences, oeis.org

[159] R. P. Stanley, Bijective proof problems (2009), 70 pp.; available at https://tinyurl.com/6avsblk

[160] R. P. Stanley, Enumerative Combinatorics, vol. 1 (second ed.) and vol. 2, Cambridge Univ. Press, 2012 and 1999

[161] R. P. Stanley, D-finiteness of certain series associated with group algebras, in Oberwolfach Rep. 11 (2014), p. 708; available at http://tinyurl.com/lza6v2e

[162] R. P. Stanley, Catalan numbers, Cambridge Univ. Press, New York, 2015.

[163] E. Steingrímsson, Some open problems on permutation patterns, in Surveys in combinatorics, Cambridge Univ. Press, Cambridge, 2013, 239-263.

[164] J. J. Sylvester, Proof of the hitherto undemonstrated Fundamental Theorem of Invariants, Philosophical Magazine 5 (1878), 178-188; available at http://tinyurl.com/c94pphj.

[165] T. Tao, E. Croot and H. Helfgott, Deterministic methods to find primes, Math. Comp. 81 (2012), 12331246.

[166] A. G. Thomason, Hamilton cycles and uniquely edge-colourable graphs, Ann. Discrete Math. 3 (1978), 259-268.

[167] W. T. Tutte, On Hamiltonian circuits, J. LMS 21, (1946), 98-101.

[168] W. T. Tutte, Graph theory as I have known it, Oxford Univ. Press, New York, 1998. 
[169] L. G. Valiant, Completeness classes in algebra, in Proc. 11th STOC (1979), ACM, New York, $249-261$.

[170] V. Vatter, Permutation Classes, in The Handbook of enumerative combinatorics, CRC Press, Boca Raton, FL, 2015, 753-834.

[171] S. O. Warnaar and W. Zudilin, A q-rious positivity, Aeq. Math. 81 (2011), 177-183.

[172] J. West, Generating trees and the Catalan and Schröder numbers, Discrete Math. 146 (1995), $247-262$.

[173] A. Wigderson, Mathematics and Computation, book draft (Jan. 2018), to be published by Princeton Univ. Press, Princeton, NJ; available at https://www.math.ias.edu/avi/book

[174] H. S. Wilf, What is an answer?, Amer. Math. Monthly 89 (1982), no. 5, 289-292.

[175] D. B. Wilson, Generating random spanning trees more quickly than the cover time, in Proc. 28th STOC (1996), ACM, New York, 296-303.

[176] W. Woess, Random walks on infinite graphs and groups, Cambridge U. Press, Cambridge, 2000.

[177] J. Yang, Rectangular tileability and complementary tileability are undecidable, Eur. J. Combin. 41 (2014), 20-34.

[178] D. Zagier, Elliptic modular forms and their applications, in The 1-2-3 of modular forms, Springer, Berlin, 2008, 1-103.

[179] D. Zeilberger, Enumerative and Algebraic Combinatorics, in Princeton companion to mathematics, (T. Gowers, J. Barrow-Green and I. Leader, eds.), Princeton Univ. Press, Princeton, NJ, 2008. 